\title{
Histone deacetylase 9 promotes endothelial- mesenchymal transition and an unfavorable atherosclerotic plaque phenotype
}

\author{
Laura Lecce, ${ }^{1}$ Yang Xu, ${ }^{1}$ Bhargavi V'Cangula, ${ }^{1}$ Nirupama Chandel, ${ }^{1}$ Venu Pothula, ${ }^{1}$ Axelle Caudrillier, ${ }^{2}$ Maria Paola Santini, ${ }^{1}$ \\ Valentina d'Escamard, ${ }^{1}$ Delaine K. Ceholski, ${ }^{1}$ Przemek A. Corski, ${ }^{1}$ Lijiang Ma, ${ }^{1,3}$ Simon Koplev, ${ }^{3}$ Martin Mæng Bjørklund, ${ }^{4,5}$ \\ Johan L.M. Björkegren, ${ }^{3,6}$ Manfred Boehm, ${ }^{7}$ Jacob Fog Bentzon, ${ }^{4,5}$ Valentin Fuster, ${ }^{1,5}$ Ha Won Kim, ${ }^{8}$ Neal L. Weintraub, ${ }^{8}$ \\ Andrew H. Baker, ${ }^{2}$ Emily Bernstein, ${ }^{9}$ and Jason C. Kovacic ${ }^{1,10,11}$
}

${ }^{1}$ Cardiovascular Research Institute, Icahn School of Medicine at Mount Sinai, New York, New York, USA. ${ }^{2}$ Centre for Cardiovascular Science, University of Edinburgh, Edinburgh, United Kingdom. ${ }^{3}$ Department of Genetics and Genomic Sciences, Icahn School of Medicine at Mount Sinai, New York, New York, USA. ${ }^{4}$ Department of Clinical Medicine, Heart Diseases, Aarhus University, Aarhus, Denmark. ${ }^{5}$ Centro Nacional de Investigaciones Cardiovasculares (CNIC), Madrid, Spain. ' Integrated Cardio Metabolic Centre, Department of Medicine, Karolinska Institutet, Karolinska Universitetssjukhuset, Huddinge, Sweden. 'Laboratory of Cardiovascular Regenerative Medicine, Translational Vascular Medicine Branch, National Heart Lung and Blood Institute, NIH, Bethesda, Maryland, USA. ${ }^{8}$ Department of Medicine, Cardiology Division and Vascular Biology Center, Medical College of Georgia at Augusta University, Augusta, Georgia, USA. ${ }^{9}$ Departments of Oncological Sciences and Dermatology, Icahn School of Medicine at Mount Sinai, New York, New York, USA. ${ }^{10}$ Victor Chang Cardiac Research Institute, Darlinghurst, New South Wales, Australia. "St. Vincent's Clinical School, University of New South Wales, Sydney, Australia.

Endothelial-mesenchymal transition (EndMT) is associated with various cardiovascular diseases and in particular with atherosclerosis and plaque instability. However, the molecular pathways that govern EndMT are poorly defined. Specifically, the role of epigenetic factors and histone deacetylases (HDACs) in controlling EndMT and the atherosclerotic plaque phenotype remains unclear. Here, we identified histone deacetylation, specifically that mediated by HDAC9 (a class Ila HDAC), as playing an important role in both EndMT and atherosclerosis. Using in vitro models, we found class Ila HDAC inhibition sustained the expression of endothelial proteins and mitigated the increase in mesenchymal proteins, effectively blocking EndMT. Similarly, ex vivo genetic knockout of Hdac 9 in endothelial cells prevented EndMT and preserved a more endothelial-like phenotype. In vivo, atherosclerosis-prone mice with endothelial-specific Hdac9 knockout showed reduced EndMT and significantly reduced plaque area. Furthermore, these mice displayed a more favorable plaque phenotype, with reduced plaque lipid content and increased fibrous cap thickness. Together, these findings indicate that HDAC9 contributes to vascular pathology by promoting EndMT. Our study provides evidence for a pathological link among EndMT, HDAC9, and atherosclerosis and suggests that targeting of HDAC9 may be beneficial for plaque stabilization or slowing the progression of atherosclerotic disease.

\section{Introduction}

Endothelial-mesenchymal transition (EndMT) is an essential process during cardiac development, contributing to cardiac valve formation and the stabilization of new vasculature in the developing embryo (1,2). EndMT also plays a key role in adult tissue regeneration and wound healing, in which endothelial cells acquire fibroblast-like functions to participate in extracellular matrix remodeling and tissue rebuilding $(1,2)$. Under certain pathological conditions, EndMT contributes to the progression of chronic fibrosing states (3) and is reported to occur during pulmonary (4, $5)$, renal $(6,7)$, and cardiac fibrosis $(8,9)$. EndMT also participates in vascular remodeling and neointima formation, for example, as

Authorship note: LL and YX contributed equally to this work.

Conflict of interest: The authors have declared that no conflict of interest exists. Copyright: ( 2021 , Lecce et al. This is an open access article published under the terms of the Creative Commons Attribution 4.0 International License.

Submitted: July 26, 2019; Accepted: June 11, 2021; Published: August 2, 2021.

Reference information: J Clin Invest. 2021;131(15):e131178.

https://doi.org/10.1172/JCI131178. a response to vein graft transplantation into the arterial circulation (10). More recently, it was shown that EndMT is associated with atherosclerotic plaque burden (11) and an unstable plaque phenotype in both mice and humans $(12,13)$.

EndMT can be induced by a variety of stimuli, such as exposure to cytokines, growth factors, oxidative stress, and inflammatory signals. Several signaling pathways have been associated with EndMT, most notably the TGF- $\beta$ pathway $(2,14)$. Conversely, the FGF pathway is known to inhibit EndMT by maintaining expression of EndMT-inhibitory miRNAs, which belong to the let-7 miRNA family and the miRNA-17-92 cluster (15-17). Of interest, a recent study showed that the long noncoding antisense transcript of GATA6 regulates EndMT via downstream changes in histone methylation (18). Beyond this, however, little is known about the biologic pathways and signaling mechanisms governing EndMT and the higher-order regulators at the apex of these EndMT pathways (1).

Epigenetic mechanisms and regulators of posttranslational modifications (PTMs), such as acetylation and methylation, are known to govern epithelial-to-mesenchymal transition (EMT), 
which is closely related to EndMT (19-22). Acetylation is carefully controlled through a balance of histone deacetylases (HDACs) and histone acetyltransferases (HATs), which have antagonistic functions. HDACs are a family of enzymes categorized into 5 main groups, class I (HDAC1, -2, -3, -8), class IIa (HDAC4, -5, -7, -9), class IIb (HDAC6, -10), class III (Sirt1-7), and class IV (HDAC11), that are responsible for removing acetyl groups from histones and nonhistone proteins $(23,24)$. Aberrant HDAC expression and activity can promote EMT and cancer metastasis, while HDAC inhibitors can prevent EMT (25-27). Because EndMT is a specific form of EMT $(1,2)$, this provides a strong rationale for believing that HDACs may also be of importance in regulating EndMT. Changes in HDAC expression and the use of HDAC inhibitors have also been suggested as modulating the progression of atherosclerosis and other vascular diseases by regulating macrophage cholesterol efflux and vascular smooth muscle cell proliferation $(28,29)$. Furthermore, HDACs have been implicated in a range of additional cardiovascular diseases, including pulmonary artery hypertension (30), reperfusion injury and fibrosis (31), cardiac hypertrophy and heart failure (32), stroke (33), neointima formation $(34,35)$, and abdominal aortic aneurysm (36).

Based on this body of data, we speculated that changes in histone PTMs and HDACs may govern EndMT. Furthermore, we hypothesized that by modulating EndMT, HDACs may participate in the maintenance of endothelial cell homeostasis and also in the progression of atherosclerosis and plaque instability.

\section{Results}

EndMT is associated with deacetylation of histone $H 3$ lysine residues and increased HDAC9 expression. We used a previously published method (12) of EndMT induction on 8 lines of human coronary artery endothelial cells (HCAECs) (from 8 different donors). We used HCAECs because these are the most relevant cells for coronary artery disease (CAD). A combination of TGF- $\beta 2$ plus $\mathrm{H}_{2} \mathrm{O}_{2}$ was more potent in inducing EndMT in HCAECs than either agent alone and resulted in significant downregulation of endothelial genes, including zona occludens 2 (ZO2), intercellular adhesion molecule 2 (ICAM2), occludin, and endothelial nitric oxide synthase (eNOS) (Supplemental Figure 1A; supplemental material available online with this article; https://doi.org/10.1172/ JCI131178DS1), with upregulation of mesenchymal genes transgelin $(S M 22 \alpha)$, fibroblast activation protein $(F A P)$, versican, and calponin (Supplemental Figure 1B). EndMT induction also resulted in an upregulation of TGF- $\beta$ pathway-associated transcription factors SNAIL and SLUG (Supplemental Figure 1C). These results are similar to what was seen in previously reported findings using venous endothelial cells (12).

To study relevant epigenetic effects, we first sought to characterize broad patterns of histone PTMs during EndMT. Chromatin was extracted from HCAECs after EndMT induction and screened for the abundance of common histone PTMs compared with control cells. This demonstrated that EndMT is associated with a global decrease in histone PTMs that are associated with gene activation, specifically $\mathrm{H} 3 \mathrm{~K} 4 \mathrm{me} 3 \mathrm{H} 3 \mathrm{~K} 9 \mathrm{ac}$, and H3K27ac, with a concomitant increase in H3K27me3 (Figure 1A). Since HDACs are responsible for the deacetylation of histone residues, gene expression levels of class I and II HDACs were measured using quantitative real-time PCR (qRT-PCR) at 24 hours and 5 days after EndMT induction. The mRNA expression of class IIa HDACs was most significantly altered during EndMT, with a 2-fold increase in HDAC9 and a reduction in HDAC5 and -7 (Figure 1B). Conversely, the expression of class I and IIb HDACs remained unchanged during EndMT, suggesting that class IIa HDACs may play a role in EndMT. Because we observed a generalized reduction of histone acetylation with EndMT (Figure 1A) and HDAC9 was the only HDAC to fit this pattern (increased HDAC9 expression with EndMT; Figure 1B), we reasoned that HDAC9 may play an important function in EndMT and elected to pursue its potential role in this process.

Class IIa HDAC inhibition prevents HDAC9 upregulation and H3 deacetylation during EndMT. To investigate the potential role of class IIa HDACs in EndMT, we used the chemical HDAC inhibitor MC1568 $(37,38)$ during EndMT induction. MC1568 is a selective inhibitor of class IIa HDACs and is reported to have greater than 170-fold selectivity over class I HDACs, including HDAC1 (38-40). In addition, as well as inhibiting class IIa HDAC activity, MC1568 has been shown to inhibit class IIa HDAC expression $(41,42)$.

EndMT caused changes in HCAEC morphology and reduced cell confluency, which were dose dependently prevented by increasing concentrations of MC1568 (Figure 1C). With respect to the effect of MC1568 on class IIa HDAC levels, MC1568 dose dependently prevented the EndMT-induced increase in HDAC 9 mRNA (Figure 1D). MC1568 also prevented the EndMT-associated increase in HDAC9 protein expression, as shown by both Western blotting (Figure 1E) and immunofluorescence staining (Figure 1F). MC1568 treatment during EndMT did not alter the mRNA expression of any other class IIa HDACs (Figure 1D) or HDACs from class I and class IIb (Supplemental Figure 1, D and E). Interestingly, unlike HDAC9 and in the opposite direction of the generalized reduction of histone acetylation with EndMT (Figure 1A), protein expression of HDAC4 and -7 was significantly reduced following EndMT induction, and this remained unchanged in the presence of MC1568 (Figure 1E). Addition of $7 \mu \mathrm{M}$ MC1568 prevented the EndMT-associated reduction in $\mathrm{H} 3 \mathrm{~K} 9$ and $\mathrm{H} 3 \mathrm{~K} 27$ acetylation and also prevented the increase in H3K27 methylation (Supplemental Figure 1, F and G). This dose of $7 \mu \mathrm{M}$ MC1568 was chosen based on a dose response in which this was the highest dose of MC1568 for which no cell death was observed (results not shown).

Class IIa HDACs are known to shuttle between the nucleus and cytoplasm depending on their activation state and whether or not they are targeting histones or cytoplasmic proteins. Therefore, subcellular fractionation was performed to investigate the localization of HDAC9 during EndMT and with MC1568. We identified both nuclear and cytoplasmic HDAC9 and showed that the EndMT-induced increase in HDAC9 protein primarily occurs in the cytoplasmic fraction and is prevented by MC1568 (Supplemental Figure 2A). Since histone acetylation can also be altered via HATs, we evaluated the protein expression of 2 key HATs: P300 and P300/CBP-associated factor (PCAF). Both P300 and PCAF protein expression were significantly reduced with EndMT, and while the reduction of PCAF was prevented with MC1568, P300 remained significantly downregulated (Supplemental Figure 2B). This suggests that while the deacetylation associated with EndMT is likely due to increased HDAC9, restoration of acetylation in the 
A
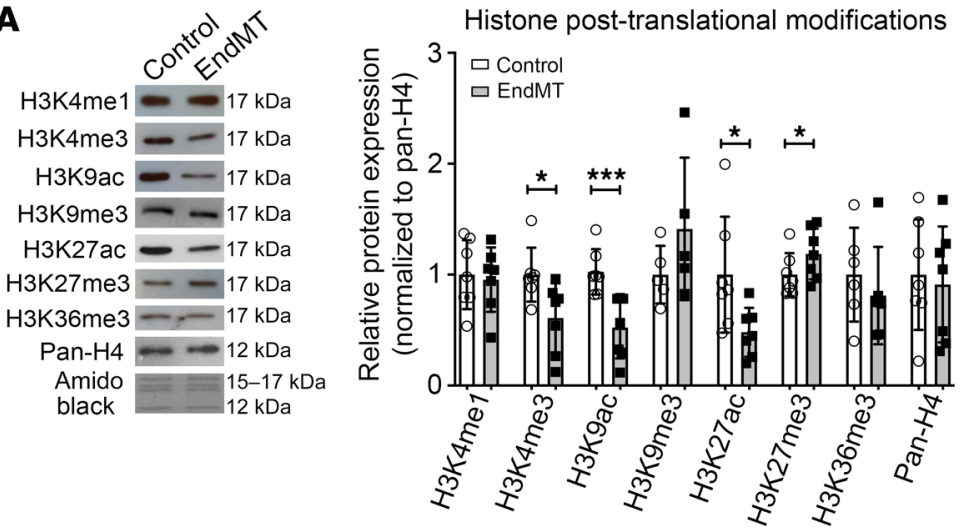

C
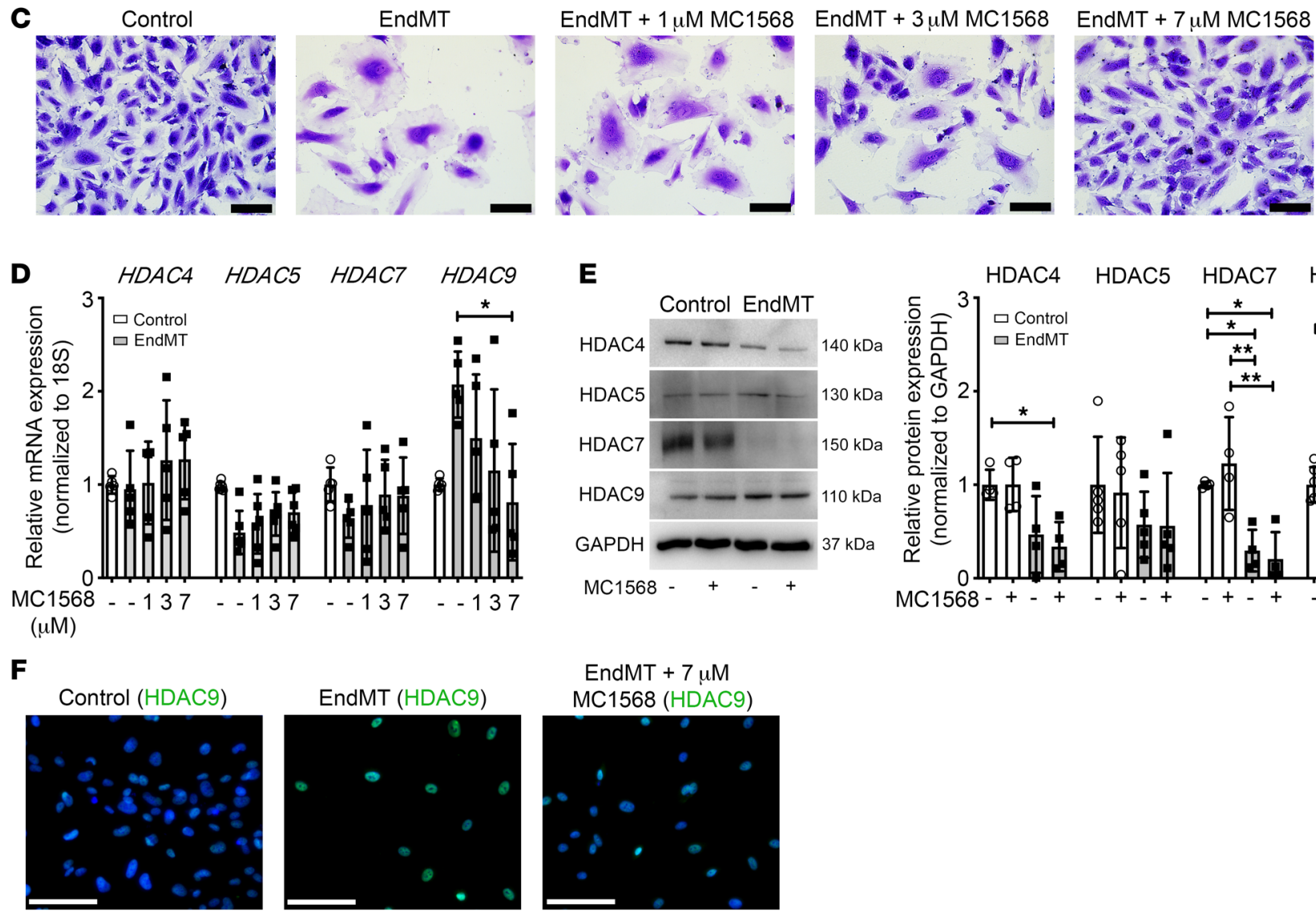

HDAC7 HDAC9

E
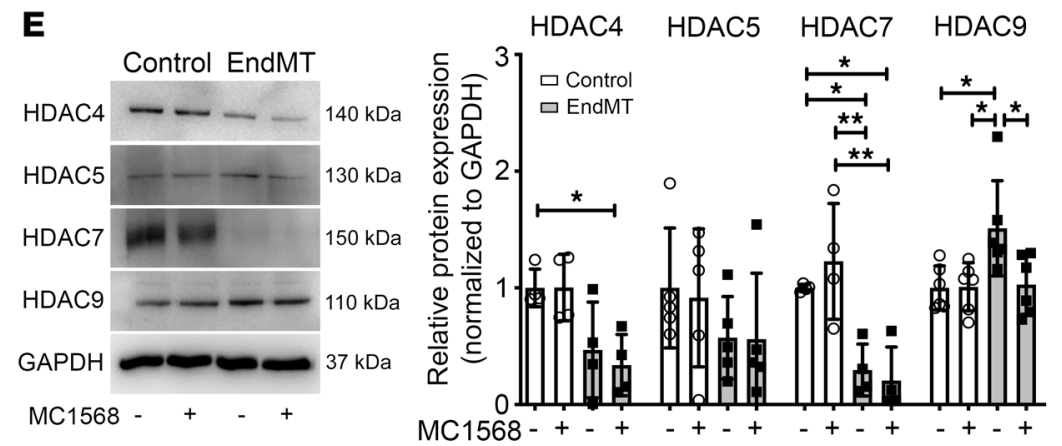

Figure 1. EndMT is associated with histone deacetylation and increased HDAC9 expression, which is ameliorated by class lla HDAC inhibitor MC1568. (A) Representative Western blots for histone PTMs after 5 days of EndMT induction in HCAECs with densitometric analysis demonstrating changes in histone acetylation and methylation. $n=5-7$. (B) qRT-PCR showing time-dependent changes of canonical HDACs after 24-hour and 5-day EndMT induction (TCF- $\beta 2$ plus $\mathrm{H}_{2} \mathrm{O}_{2}$ ) in HCAECs. Graph is representative of fold change relative to vehicle-treated control cells normalized to 1 (dashed line). $n=4-6$. (C) Images of HCAECs after 5-day EndMT induction with or without increasing doses of MC1568. Scale bars: $100 \mu \mathrm{m}$. (D) qRT-PCR analysis showing mRNA expression of class Ila HDACs (HDAC4, -5, -7, and -9) after 5-day EndMT induction in HCAECs, with a dose-dependent effect of MC1568 on HDAC9 gene expression. $n=5-6$. (E) Representative Western blots and densitometry measurements of class Ila HDACs after 5-day EndMT induction with or without MC1568 in HCAECs, with the graph representing fold change relative to vehicle-treated controls. $n=4-6$. (F) Immunofluorescence staining of control HUVECs with anti-HDAC9 antibody and after 5 -day EndMT induction with or without $7 \mu \mathrm{M}$ MC1568. Scale bars: $100 \mu \mathrm{m} .{ }^{*} P \leq 0.05 ;{ }^{* *} P \leq 0.01 ;{ }^{* * *} P \leq 0.001$. Analyses performed using unpaired Student's $t$ test (A), 1-way ANOVA (B and D), and 2-way ANOVA (E).

presence of the class IIa HDAC inhibitor can be due to a lack of combined HDACs as well as the action of HATs.

Class IIa HDAC inhibition affects EndMT-associated gene and protein expression. Since MC1568 prevented many of the EndMTinduced changes in epigenetic regulators of acetylation, the effect of MC1568 on classical EndMT-associated genes and proteins was then examined. While we observed no effect on endothelial gene expression with MC1568 in the setting of EndMT (Figure 2A), MC1568 prevented EndMT-associated increases in SM22 $\alpha$ and FAP mRNA expression in a dose-dependent manner (Figure 2B) 
A

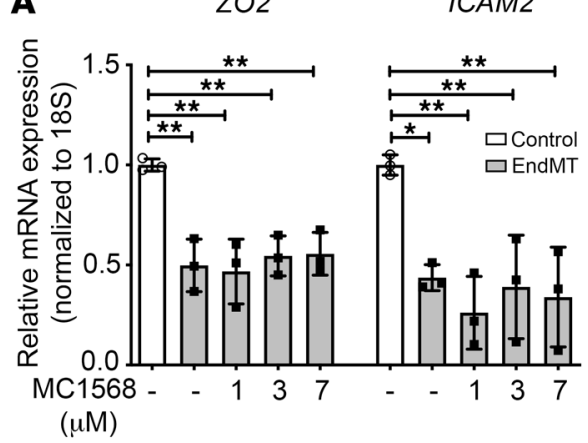

B

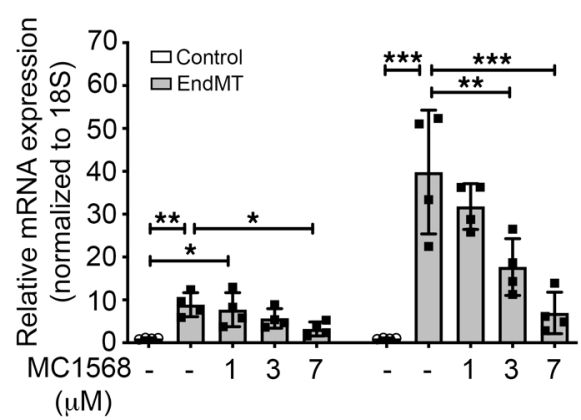

C

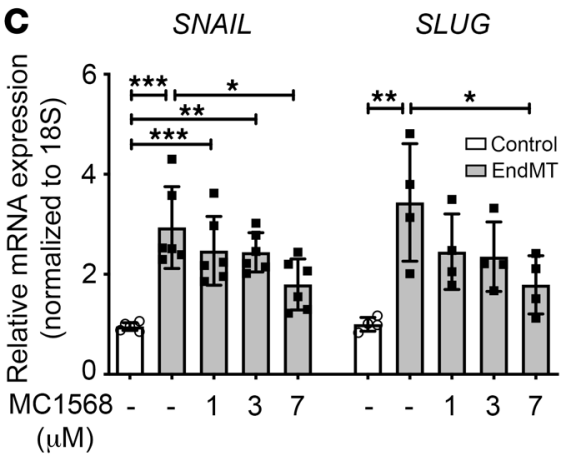

D
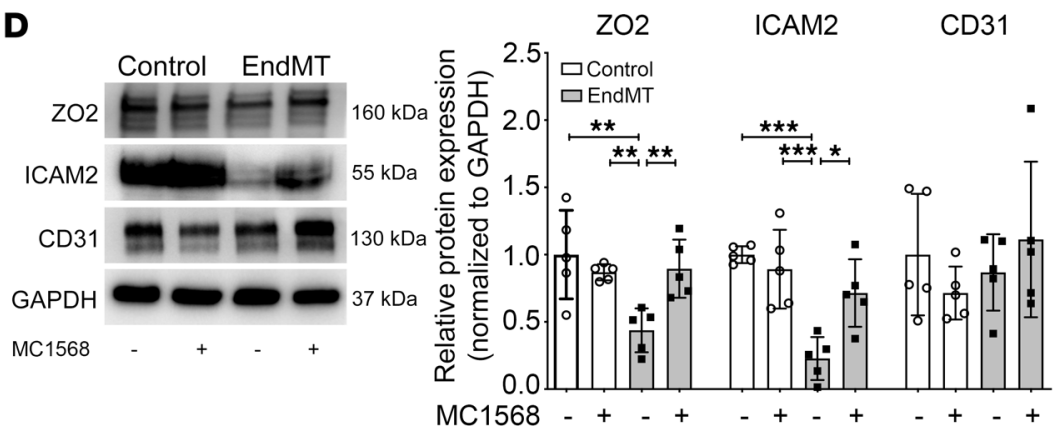

$\mathbf{E}$
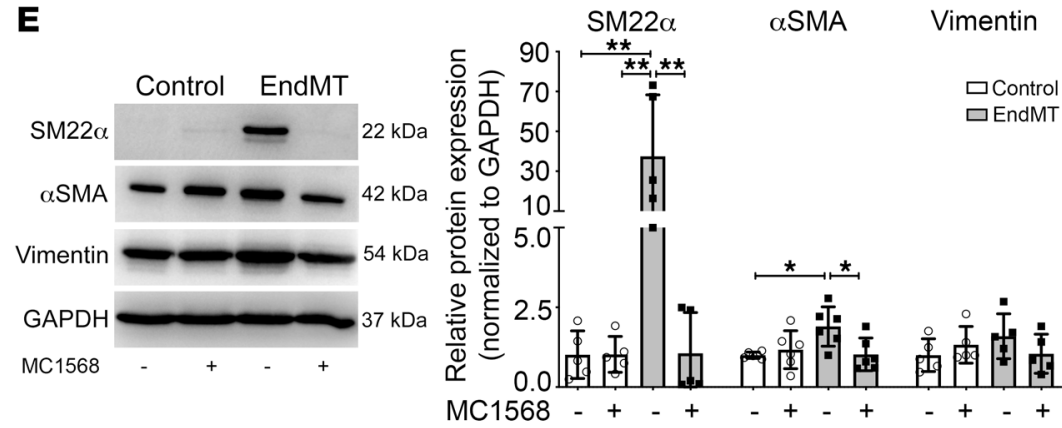

$\mathbf{F}$
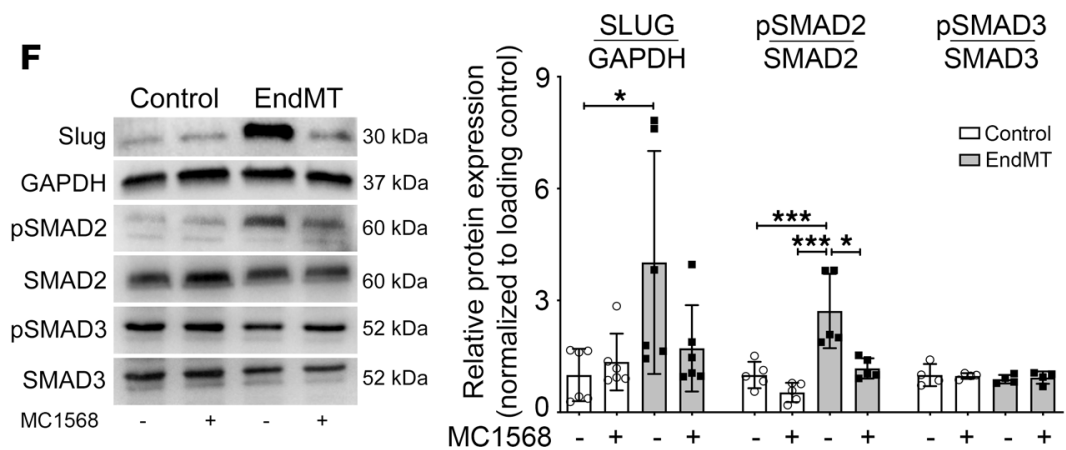

Figure 2. Class Ila HDAC inhibition suppresses EndMT-associated changes in mRNA and protein levels. (A-C) qRT-PCR analysis of endothelial (ZO2, ICAM2; $n=3$ ), mesenchymal (SM22 $\alpha, F A P ; n=4)$, and TCF- $\beta$ pathway (SNAIL, SLUG; $n=4-6)$ transcript levels after 5-day EndMT induction with or without increasing doses of MC1568 in HCAECs. (D and E) Representative Western blots and densitometry measurements of endothelial (ZO2, ICAM2, CD31) and

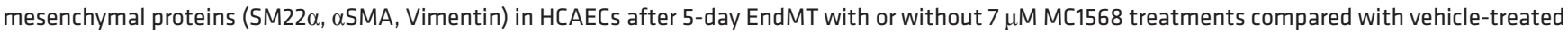
controls. $n=5-6$. (F) Representative Western blots and densitometry measurements of TGF- $\beta$-associated transcription factor proteins (SLUG, pSMAD2, pSMAD3) in HCAECs after 5-day EndMT with or without $7 \mu \mathrm{M}$ MC1568 treatment compared with vehicle-treated controls. $n=4-6 .{ }^{*} P \leq 0.05$; ${ }^{* *} P \leq 0.01$; ${ }^{* *} P \leq 0.001$. Analyses performed using 1-way ANOVA (A-C) and 2-way ANOVA (D-F).

and also regulated the TGF- $\beta$ pathway by inhibiting EndMT-associated increases in SNAIL and SLUG (Figure 2C). Interestingly, despite no effect on endothelial gene expression, MC1568 prevented the EndMT-associated decline in endothelial proteins $\mathrm{ZO} 2$ and ICAM2 (Figure 2D). In terms of mesenchymal protein upregulation with EndMT, MC1568 abolished the EndMT-associated increase in SM22 $\alpha$ and $\alpha$ SMA protein expression with a modest effect on vimentin (Figure 2E). MC1568 also prevented EndMT-associated increases in SLUG protein and the increased phosphorylation of SMAD2 (Figure 2F). Furthermore, MC1568 prevented the EndMT-associated increase in $\mathrm{FAP}^{+}$endothelial cells, as assessed by flow cytometry (Supplemental Figure 2C). Collectively, these results demonstrate that class IIa HDACs are responsible for regulating many of the widespread molecular and genetic changes that occur during EndMT.

Given that all observed EndMT-associated changes were reversed by MC1568 except for ZO2 and ICAM2 mRNA expression (Figure 2A), ChIP-quantitative PCR (ChIP-qPCR) was performed to elucidate the underlying epigenetic mechanisms. ChIP for H3K27ac with subsequent qPCR showed that at the promoter regions of both ZO2 and ICAM2, H3K27ac enrichment was dramatically decreased upon induction of EndMT, which remained unrestored in the presence of MC1568 (Supplemental Figure 2D). As a positive control for ChIP-qPCR, H3K27ac enrichment at the FAP promoter was also examined and exhibited an increase during EndMT induction, which was partially prevented by MC1568. 
A

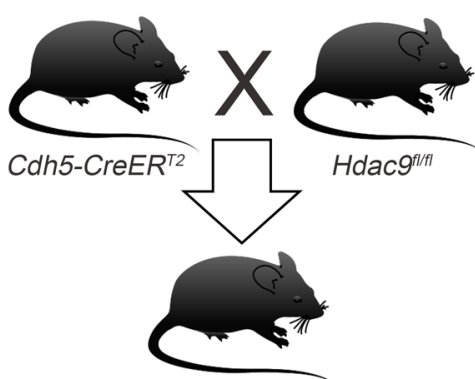

Cdh5-CreER ${ }^{T 2} ;$ Hdac $^{\text {II/ }}$

(Endo-Hdac9ko)
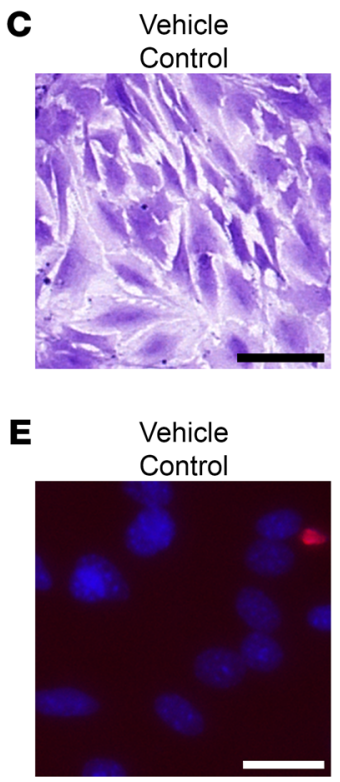

$\mathbf{F}$

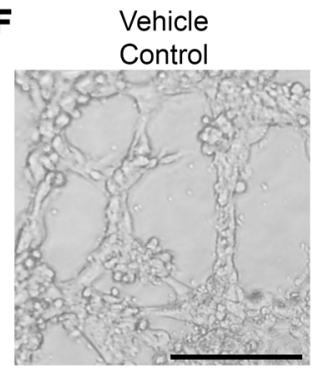

G

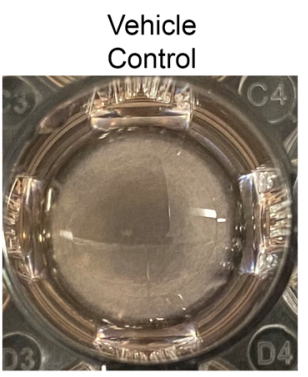

Control
Endo-Hdacgko Control

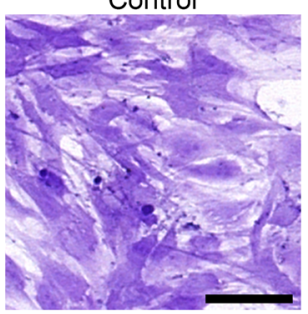

\section{Endo-Hdac9ko}

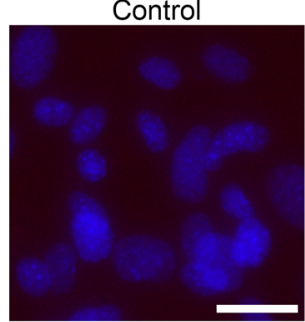

Endo-Hdac9ko

Control

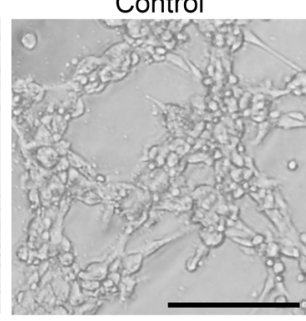

Endo-Hdac9ko

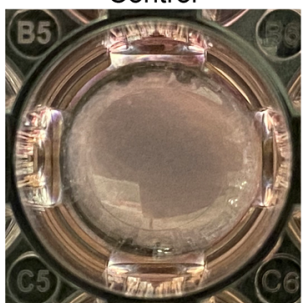

B

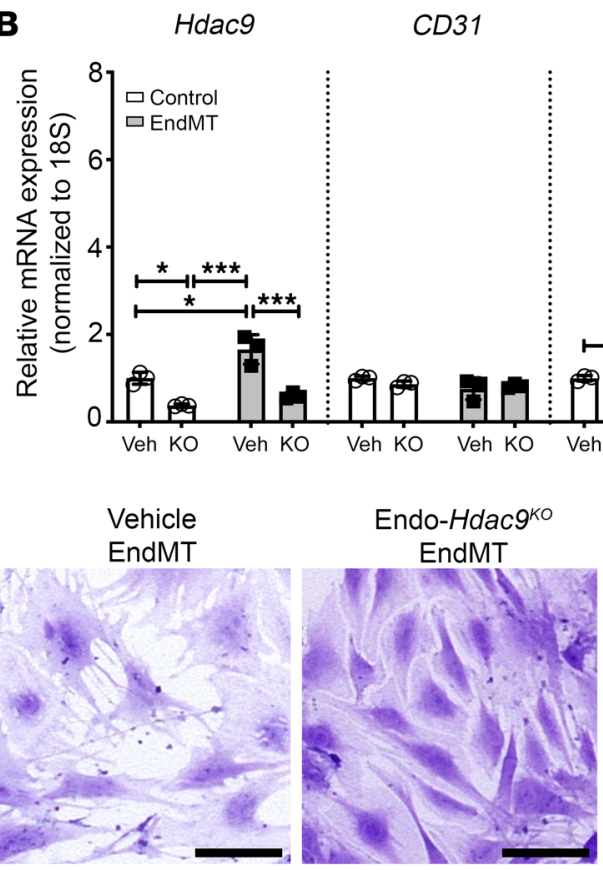

Endo-Hdacgko
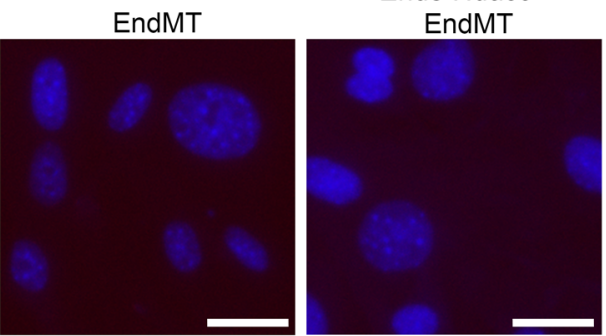

Endo-Hdac $9^{k o}$

EndMT
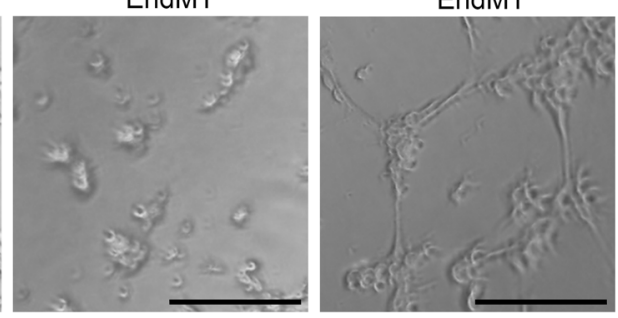

Endo-Hdac9ko EndMT
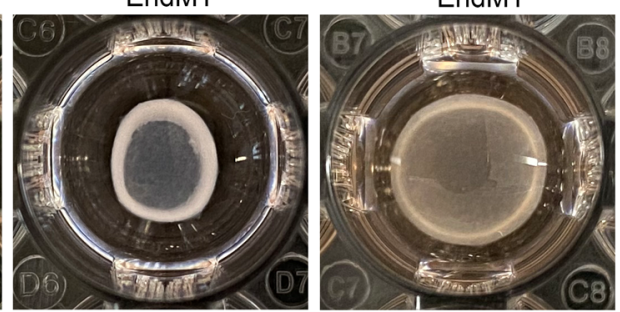

Icam2

$\operatorname{sm} 22 \alpha$
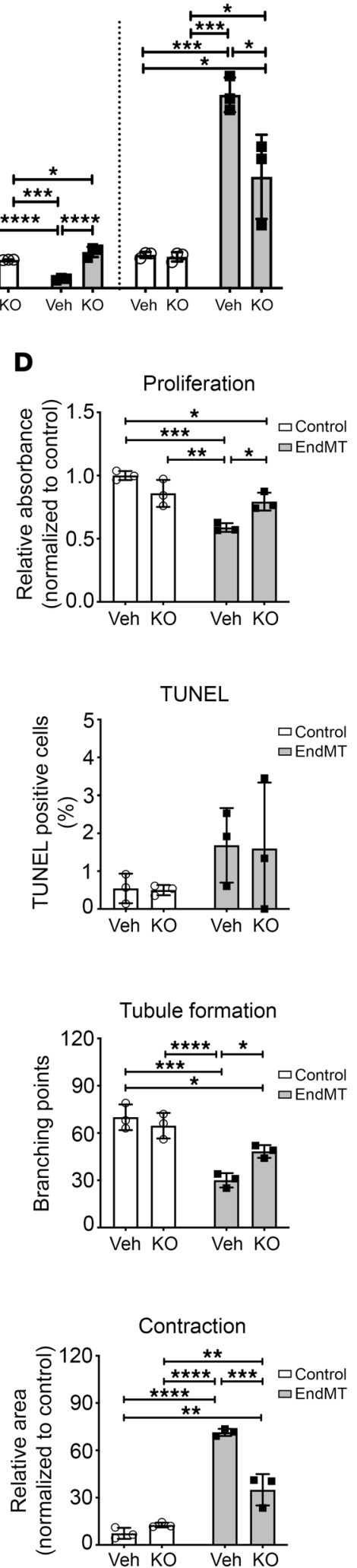
Figure 3. Ex vivo endothelial-specific $\mathrm{Hdac9}$ knockout attenuates EndMT-associated changes in endothelial cell function in MPLECs. (A) Breeding and generation of Endo-Hdac ${ }^{K O}$ mice for obtaining MPLECs. (B) Expression of Hdac9, CD31, Icam2, and Sm22 $\alpha$ in MPLECs with (KO; i.e., ex vivo 4-OH tamoxifen treatment) and without (Veh; vehicle treatment) knockout of Hdac9 with or without EndMT assessed by qRT-PCR. These groups are identical for all subsequent panels in this figure. (C) Crystal violet staining showing changes in cell numbers and density. Scale bars: $100 \mu \mathrm{m}$. (D) To assess proliferation, MPLECs were incubated with BrdU, followed by spectrophotometric quantification. Data are represented as fold change compared with vehicle-treated control cells. (E) Representative images and quantification of TUNEL assay to detect apoptosis on MPLECs with or without EndMT induction. Scale bars: $30 \mu \mathrm{m}$. (F) Tubule formation of MPLECs with or without EndMT assessed by plating cells onto Matrigel and incubating for another 4 hours. Tubule branch points were imaged and quantified. Scale bars: $100 \mu \mathrm{m}$. (G) Contraction assay showing changes in relative unoccupied area (normalized to a completely unoccupied well) for MPLECs with or without EndMT induction. For this figure, lungs from $n=4$ male Endo-Hdac ${ }^{K 0}$ mice were pooled to derive MPLECs. Apart from (crystal violet staining) (C), $n=3$ for all analyses as biological replicates. ${ }^{*} P \leq 0.05 ;{ }^{* *} P \leq 0.01 ;{ }^{* * *} P \leq 0.001 ;{ }^{* * *} P \leq 0.0001$. All analyses performed using 2-way ANOVA.

ChIP-qPCR was also performed for the enrichment of H3K9ac at the ZO2 and ICAM2 promoters, which showed that $\mathrm{H} 3 \mathrm{~K} 9 \mathrm{ac}$ plays no role in regulating the expression of these 2 genes, as the enrichment of H3K9ac remained unchanged in all conditions (Supplemental Figure 2E). In contrast, H3K9ac enrichment was significantly increased at the promoter region of FAP $(P \leq 0.01$, Supplemental Figure 2E) and modestly prevented by MC1568. Together, these results confirm that MC1568 does not regulate the gene expression of endothelial markers ICAM2 and ZO2. However, these data demonstrate that increased mesenchymal (FAP) gene expression during EndMT arises in the setting of enhanced promoter acetylation, which is partially reversible by class IIa HDAC inhibition with MC1568.

To extend these findings, i.e., that class IIa HDAC inhibition with MC1568 can prevent mesenchymal, but not endothelial, gene expression changes during EndMT, we employed a different model of EndMT-induction using TGF- $\beta 2+\mathrm{IL}-1 \beta$ in HUVECs for further broad validation. Consistent with our findings in HCAECs, EndMT increased HDAC9 expression, which was prevented by the addition of MC1568 (Supplemental Figure 3A). Similarly, MC1568 was able to reverse EndMT-induced gene expression of SLUG (Supplemental Figure 3B) and mesenchymal genes (Supplemental Figure 3, F-H), while having no effect on endothelial gene expression (Supplemental Figure 3, C-E).

Class IIa HDAC inhibition prevents EndMT-induced changes in endothelial cell phenotype. Given that class IIa HDAC inhibition with MC1568 blocked EndMT-induced gene and protein changes in HCAECs in vitro, we next explored its effect on the endothelial phenotype. EndMT induction resulted in a decrease in cell proliferation, as evidenced by reduced cell density in culture and BrdU cell proliferation assay, which was partly rescued by MC1568 (Supplemental Figure 4, A and B). There was no difference in endothelial cell apoptosis with EndMT, and there was a nonsignificant reduction in apoptosis due to MC1568, although the proportion of cells undergoing apoptosis was low in all conditions (Supplemental Figure 4C). We next explored tubule-forming capacity and contraction - with tubule formation being a classical endothelial trait that is reduced with EndMT, while contraction is a mesenchymal cell trait that is enhanced by EndMT - although it is noted that these assays may not be entirely specific to EndMT $(1,43)$. Consistent with transition to a mesenchymal-like phenotype, after induction of EndMT, HCAECs exhibited decreased ability to form tubules (Supplemental Figure 4D) and increased contractility (Supplemental Figure 4E). Class IIa HDAC inhibition using MC1568 partially rescued both of these traits (Supplemental Figure 4, D and E). These results indicate that, in addition to preventing gene and protein changes, class IIa HDAC inhibition also inhibits aspects of the endothelial phenotypic and functional changes that arise due to EndMT.

Exogenous HDAC9 overexpression promotes EndMT-related changes in gene and protein expression. To confirm that HDAC9 is responsible for the effect of class IIa HDAC inhibition on EndMT and that HDAC9 has a causal relationship with EndMT-associated changes in endothelial and mesenchymal gene and protein expression, HUVECs were transfected with a plasmid (pCMV3tag6-HDAC9) containing full-length, Flag-tagged HDAC9 or empty plasmid as a control. Transfection caused an increase in transient, exogenous HDAC9 mRNA (Supplemental Figure 5A) and protein expression (Supplemental Figure 5B), which remained largely cytoplasmic (Supplemental Figure 5C). Increased HDAC9 resulted in increased expression of FAP, $\alpha S M A$, vimentin, and $S L U G$, with no effect observed on ZO2 and ICAM2 mRNA levels (Supplemental Figure 5A). Consistent with our hypothesis that HDAC9 promotes EndMT, exogenous HDAC9 caused a decrease in the protein expression of $\mathrm{ZO} 2$ and ICAM2, which was prevented by MC1568 (Supplemental Figure 5D). Collectively, these results provide further evidence that HDAC9 regulates the molecular changes associated with EndMT.

Ex vivo endothelial-specific Hdac9 knockout attenuates End$M T$. To further investigate the role of HDAC9 in EndMT, we created a mouse model with endothelial-specific Hdac9 knockout (Cdh5-CreER ${ }^{T 2}$;Hdac $9^{f / / f l}$, henceforth Endo-Hdac9KO) (Figure $3 \mathrm{~A}$ ). To first characterize the effects of endothelial-specific Hdac9 knockout at the cellular level, we cultured mouse primary lung endothelial cells (MPLECs) from Endo-Hdac9KO mice and induced Hdac9 knockout ex vivo by treatment with 4-hydroxytamoxifen (4-OH tamoxifen). Compared with what occurred in excipient-treated control cells, Hdac 9 expression was reduced by approximately 65\% in tamoxifen-treated cells (Figure 3B). Treatment with tamoxifen and ex vivo knockout of $\mathrm{Hdac} 9$ had no effect on baseline levels of CD31, Icam2, or Sm22a gene expression. However, upon induction of EndMT, the increase in $S m 22 \alpha$ and decrease in Icam2 levels were rescued in cells with knockout of Hdac9 (Figure 3B). Using the same functional assays as shown in Supplemental Figure 4 for HCAECs in vitro treated with MC1568, we observed almost identical results with MPLECs ex vivo with and without knockout of Hdac9. Specifically, EndMT induction resulted in morphological changes and a decrease in cell proliferation that was rescued by knockout of Hdac9 (Figure 3, C and D). There was no difference in endothelial cell apoptosis either with EndMT or due to Hdac9 knockout (Figure 3E). Similarly again to what is shown in Supplemental Figure 4, after induction 
A

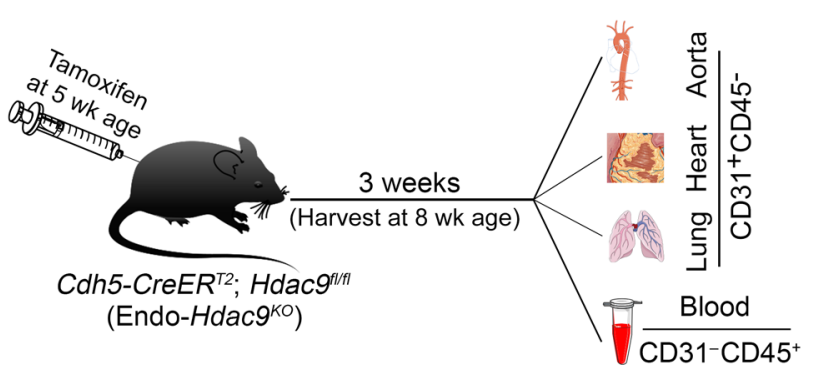

C

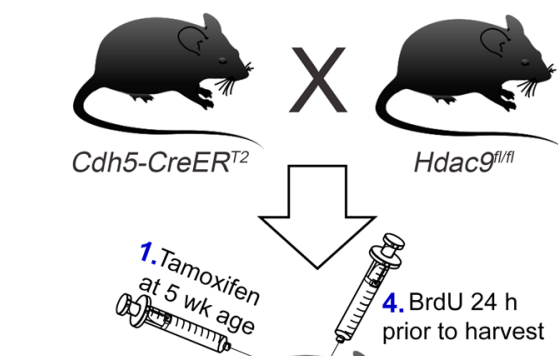

B

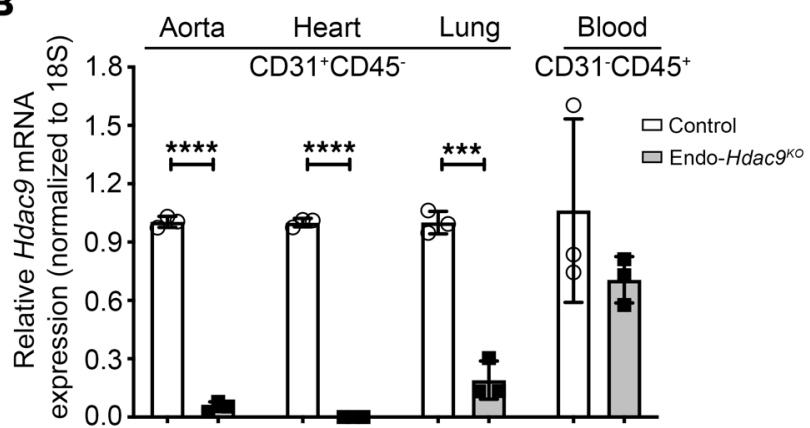

D
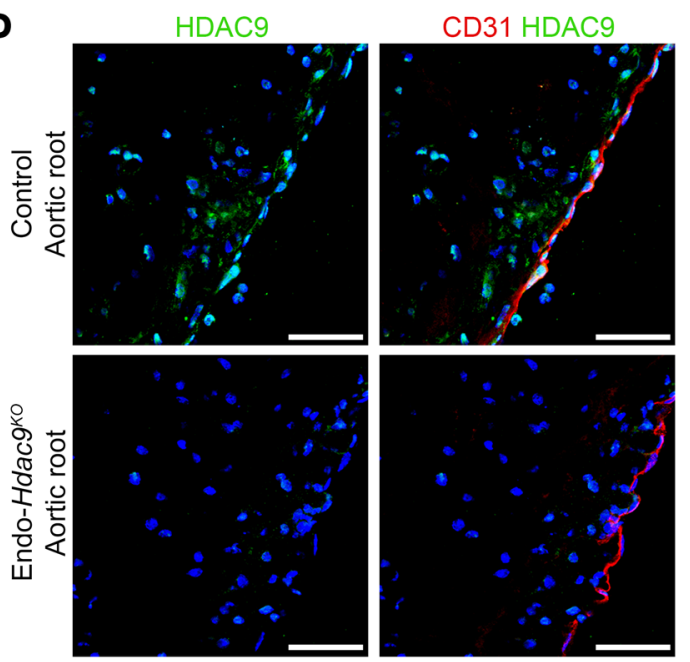

E

$\mathrm{CD} 1^{+} \alpha \mathrm{SMA}^{+} \mathrm{HDAC}^{+}$ cells/DAPI ${ }^{+}$
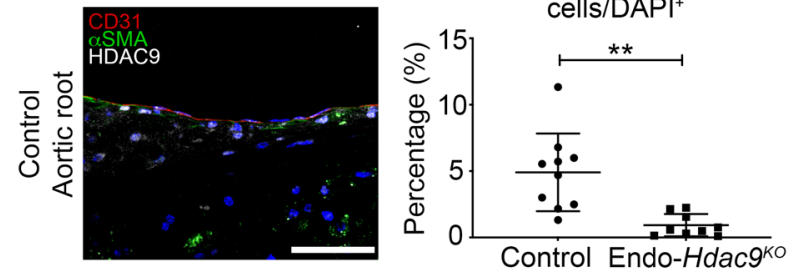

CD31+HDAC9+ cells/CD31+
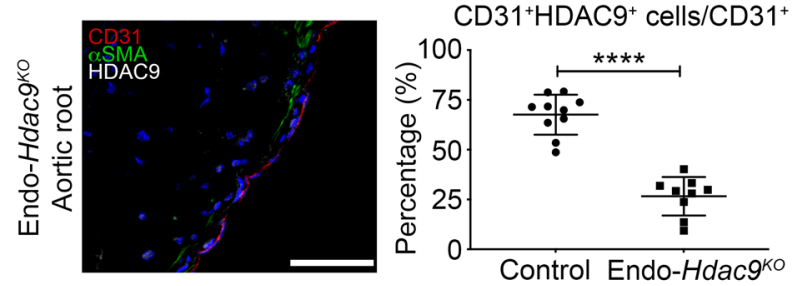

$\mathrm{CD} 1^{+} \alpha \mathrm{SMA}^{+} \mathrm{HDAC9}^{+}$ cells $/ \alpha S M A^{+} D A P I^{+}$
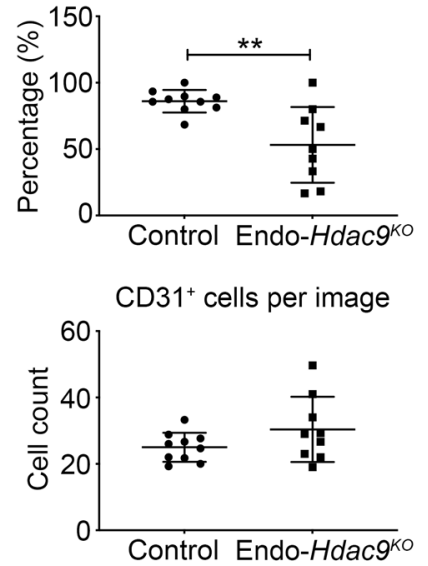

$\mathrm{CD} 1^{+} \alpha \mathrm{SMA}^{+} \mathrm{HDAC9}{ }^{+}$ cells/CD31 ${ }^{+}$

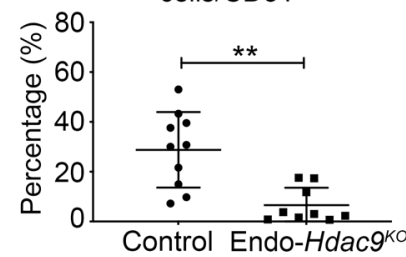

$\mathrm{DAPI}^{+}$cells per image

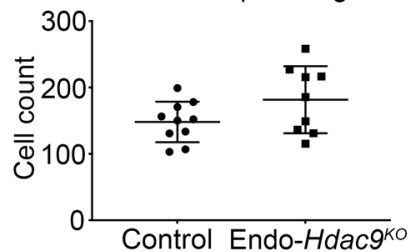

Figure 4. In vivo establishment and validation of Endo-Hdac9 ${ }^{k 0}$ mouse model. All comparisons in this figure are using endothelial-specific Hdacg

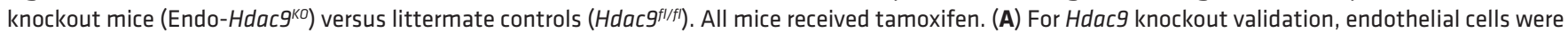
harvested from a variety of tissues from nonatherosclerotic Endo-Hdac ${ }^{\mathrm{K} 0}$ mice or littermate controls. (B) Hdac9 knockout validation: qRT-PCR analysis of the expression levels of $\mathrm{Hdac9}$ in $\mathrm{CD} 31^{+} \mathrm{CD} 45^{-}$endothelial cells from the aorta, heart, and lungs and CD $31^{-} \mathrm{CD} 45^{+}$leukocytes from blood in Endo-Hdacgko mice compared with littermate controls 3 weeks after tamoxifen administration. $n=3$. (C) Breeding and generation of atherosclerotic Endo-Hdac ${ }^{k 0}$ mouse model. (D) Representative immunofluorescence staining images for HDAC9- (green), CD31- (red), and DAPI-stained nuclei (blue) in plaques from the aortic root. (E) Representative immunofluorescence staining images for $\alpha$ SMA- (green), CD31- (red), HDAC9- (white), and DAPI-stained nuclei (blue) in aortic root plaques and quantification. Scale bars: $50 \mu \mathrm{m} . n=10$ controls versus $n=9$ Endo-Hdac $9^{K 0}$ mice. ${ }^{* *} P \leq 0.01 ;{ }^{* *} P \leq 0.001 ;{ }^{* * * *} P \leq 0.0001$. All analyses performed using unpaired Student's $t$ test except $C D 31^{+} \alpha S M A^{+} \mathrm{HDACg}^{+}$cells $/ \mathrm{CD} 31^{+}(\mathrm{E})$, for which Mann-Whitney $U$ test was used. 
A
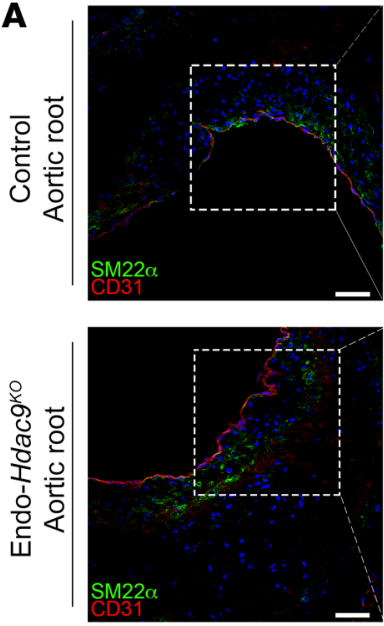

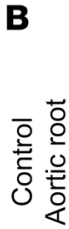
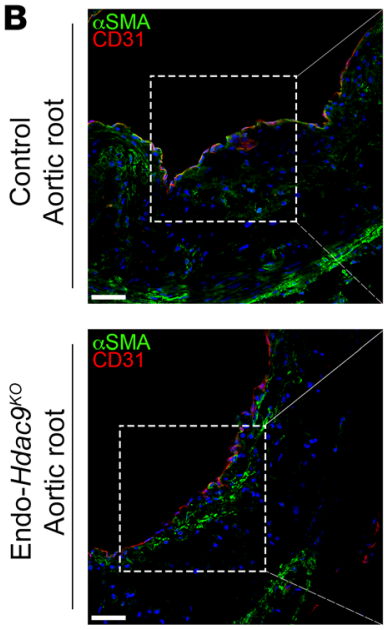

C
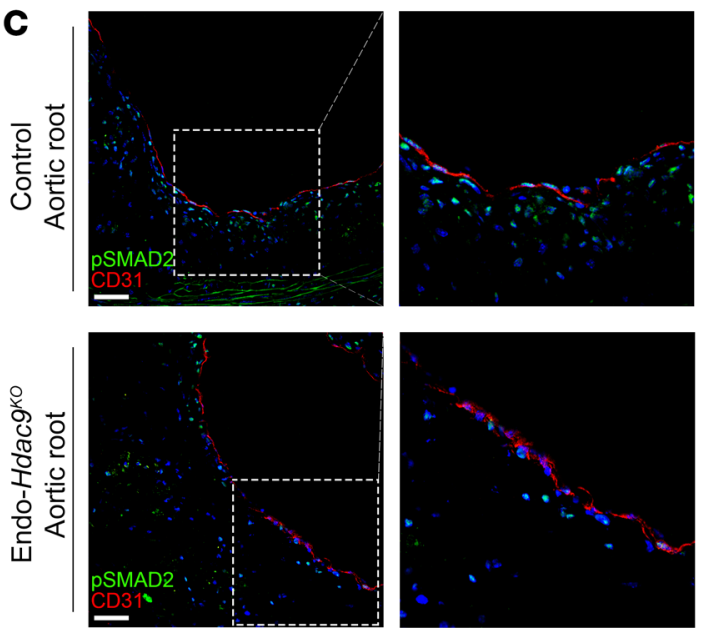

SM22 $\alpha^{+} \mathrm{CD} 31^{+}$cells/CD31
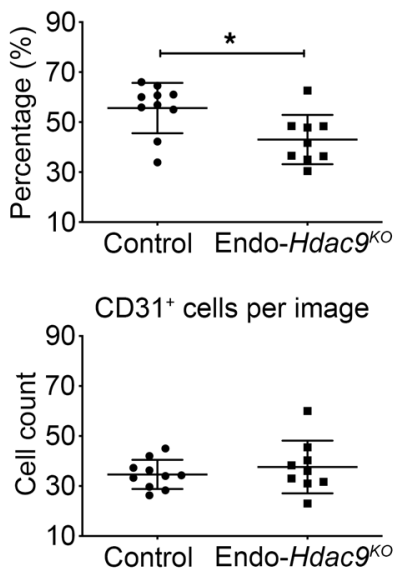

aSMA ${ }^{+} \mathrm{CD} 31^{+}$cells/CD31+
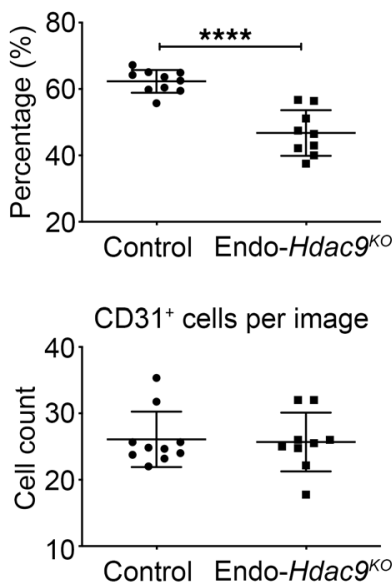

pSMAD2 ${ }^{+}$CD $31^{+}$cells/CD $31^{+}$
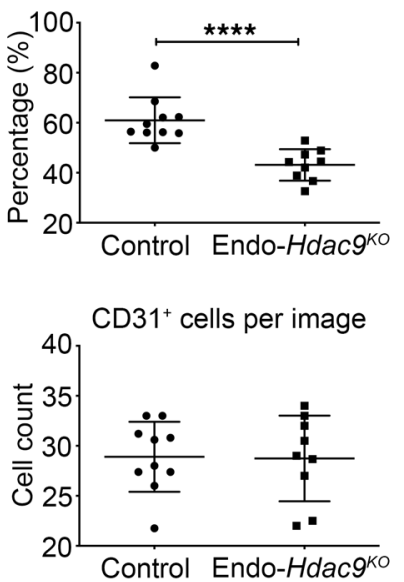

Figure 5. Endothelial-specific Hdac9 knockout reduces EndMT in vivo. All comparisons in this figure were generated using aortic root sections from Endo-Hdac ${ }^{K o}$ mice versus littermate controls, and all mice received tamoxifen. (A) Representative immunofluorescence staining images for SM22 $\alpha$ - (green), CD31- (red), and DAPI-stained nuclei (blue), quantification of SM $22 \alpha^{+}$CD $31^{+}$ copositive cells over total CD31+ cells, and total number of $\mathrm{CD} 31^{+}$cells per image. (B) Representative immunofluorescence staining images for aSMA- (green), CD31- (red), and DAPI-stained nuclei (blue), quantification of copositive cells over total $\mathrm{CD} 31^{+}$cells, and total number of $\mathrm{CD} 31^{+}$ cells per image. (C) Representative immunofluorescence staining images for pSMAD2- (green), CD31- (red), and DAPI-stained nuclei (blue), quantification of copositive cells over total $\mathrm{CD} 31^{+}$cells, and total number of CD31+ cells per image. Scale bars: $50 \mu \mathrm{m}$. Right panels are digital enlargements of the original adjacent images. $n=10$ controls versus $n=9$ Endo-Hdac ${ }^{k 0}$ mice. ${ }^{*} P \leq 0.05 ;{ }^{* * *} P$ $\leq 0.0001$. All analyses performed using unpaired Student's $t$ test except the following, for which Mann-Whitney $U$ test was used: SM22 $\alpha^{+} \mathrm{CD} 31^{+}$ cells/CD31+ cells (A); CD31+ cells per image (B); and pSMAD2 ${ }^{+}$CD $31^{+}$cells $/$CD $31^{+}$cells (C). of EndMT, MPLECs exhibited decreased ability to form tubules and showed increased contractility. Knockout of Hdac 9 partially rescued both these mesenchymal traits (Figure 3, F and G, respectively). Collectively, these findings using MPLECs with or without Hdac9 deletion confirm that HDAC9 plays a key role in governing EndMT. Moreover, the nearly identical findings shown in Supplemental Figure 4 (HCAECs undergoing EndMT with or without class IIa HDAC inhibition with MC1568) versus those in Figure 3 (MPLECs undergoing EndMT with or without Hdac9 knockout) corroborate this hypothesis.

Endothelial-specific Hdac9 knockout is associated with reduced EndMT and a favorable plaque phenotype in atherosclerosis-prone mice. In view of the above data indicating that knockout of HDAC9 inhibits EndMT as well as prior knowledge that EndMT is associat- 

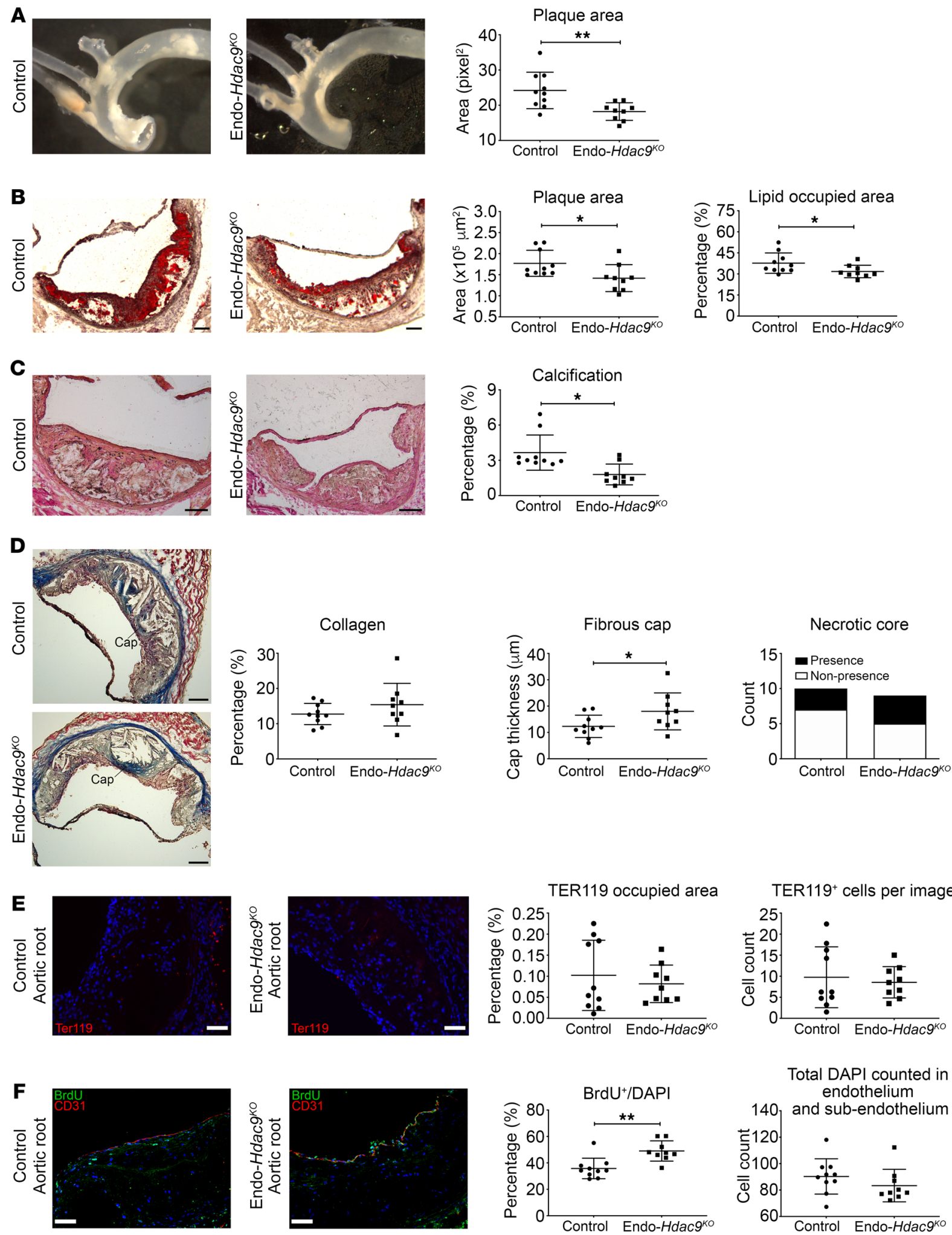

Total DAPI counted in endothelium

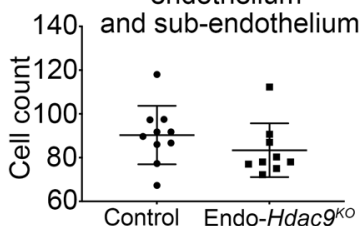


Figure 6. Endothelial-specific Hdac9 knockout reduces atherosclerosis and enhances plaque stability in vivo. All comparisons shown are using EndoHdac ${ }^{K O}$ mice versus littermate controls, and all mice received tamoxifen. (A) Representative images of aorta before embedding into OCT and en face analysis of aortic plaque. (B) Representative images of the aortic root with staining using oil red 0 , with quantification of total plaque area and plaque lipid content (red stained area as a percentage of total plaque area). (C) Representative images of aortic root sections using Von Kossa stain (black represents calcification), with quantification of calcification. (D) Representative aortic root images stained with Masson's trichrome with quantification of collagen content, fibrous cap thickness, and presence/nonpresence of necrotic core (blue, collagen; pink, macrophages and cardiac muscle). (E) Representative immunofluorescence staining images in aortic root plaques for TER119- (red, erythrocyte marker) and DAPI-stained nuclei (blue) and quantification. (F) Representative immunofluorescence staining images of aortic root plaques for BrdU- (green), CD31- (red), and DAPI-stained nuclei (blue) in the endothelial and subendothelial layers, and quantification. Scale bars: $100 \mu \mathrm{m}$ (B-D); $50 \mu \mathrm{m}$ (E and F). $n=10$ controls versus $n=9$ Endo-Hdac $\mathrm{g}^{\mathrm{KO}}$ mice for all panels. ${ }^{*} P \leq 0.05$; ${ }^{* *} P \leq 0.01$. All analyses performed using unpaired Student's $t$ test except the following, for which Mann-Whitney $U$ test was used: plaque area (B), calcification (C), and both analyses in F. In addition, presence or nonpresence of necrotic core (D) was analyzed using Fisher's exact test.

ed with atherosclerotic plaque burden (11) and an unstable plaque phenotype $(12,13)$, we investigated to determine whether HDAC9 modulates EndMT in vivo and thereby affects atherosclerosis. We leveraged our Endo-Hdac ${ }^{K O}$ mouse model (Cdh5-CreER ${ }^{T 2}$; $\left.H d a c 9^{f / f}\right)$ and used Cre-negative littermates as controls $\left(\operatorname{Hdac}^{\text {f/ff) }}\right.$. To activate Cre-mediated $H d a c 9$ deletion, all mice (Cdh5-CreER ${ }^{T 2}$; $H d a c 9^{f / f l}$ and $H d a c 9^{A / f l}$ littermate controls) received a course of tamoxifen injections beginning at 5 weeks of age. In murine tissues harvested from these mice 3 weeks after tamoxifen injections, we confirmed the effectiveness of in vivo $\mathrm{Hdac} 9$ deletion in sorted endothelial cells $\left(\mathrm{CD} 31^{+} \mathrm{CD} 45^{-}\right)$from the aorta, heart, and lungs of Endo- $H d a c 9^{K O}$ mice, whereas circulating $\mathrm{CD} 31^{-} \mathrm{CD} 45^{+}$leukocytes showed similar levels of $\mathrm{Hdac} 9$ in both groups (Figure 4, A and B).

To induce atherosclerosis in Endo-Hdac ${ }^{K O}$ mice and their littermate controls, we administered a single dose of proprotein convertase subtilisin/kexin type 9-encoding (PCSK9-encoding) recombinant adeno-associated viral vector at 7 weeks of age and then fed mice a high-fat diet from 8 weeks of age for 16 weeks. Tissues were harvested at 24 weeks of age (Figure 4C). Compared with controls, Endo-Hdac $9^{K O}$ mice showed no differences in total body weight, lipid levels, or hematological cell counts (Supplemental Figure 6, A-D). Diastolic blood pressure differed marginally between groups in 8-week-old mice, but there was no subsequent difference between these same groups for diastolic blood pressure at 16 or 24 weeks of age (Supplemental Figure 6E). There was no difference between groups for systolic blood pressure at any time point (Supplemental Figure 6E).

We next performed immunofluorescence staining followed by confocal microscopy to assess HDAC9 expression patterns in the atherosclerotic aorta. In control mice, we identified clear endothelial expression of HDAC9, which was almost undetected in Endo-Hdac ${ }^{K O}$ mice (Figure 4D). Furthermore, while there was no difference in either the total number of cells or number of $\mathrm{CD} 1^{+}$endothelial cells, compared with plaques from control mice, plaques from Endo- $H d a c 9^{K O}$ mice showed a marked reduction in the proportion of $\mathrm{CD} 31^{+}$endothelial cells that coexpressed HDAC9 $\left(\mathrm{CD} 31^{+} \mathrm{HDAC}^{+}\right)$and also a marked reduction in the proportion of $\mathrm{CD} 31^{+} \mathrm{HDAC}^{+}$cells that appeared to be undergoing EndMT, as represented by concurrent expression of aSMA $\left(\mathrm{CD}^{2} 1^{+} \mathrm{HDAC}^{+} \alpha \mathrm{SMA}^{+}\right)$(Figure $\left.4 \mathrm{E}\right)$.

We also assessed the extent of EndMT on the basis of copositive staining of the endothelial marker CD31 with either SM22a, aSMA, or pSMAD2 as mesenchymal markers. We found that the proportion of cells undergoing EndMT $\left(\mathrm{SM} 22 \alpha^{+} \mathrm{CD} 31^{+}\right.$or $\mathrm{SSMA}^{+} \mathrm{CD} 31^{+}$or $\mathrm{pSMAD}^{+} \mathrm{CD} 31^{+}$copositive cells) was signifi- cantly reduced in atherosclerotic lesions from 3 different vascular areas (aortic root, ascending aorta, aortic arch) in Endo-Hdac $9^{K O}$ mice compared with controls (Figure 5 and Supplemental Figure 7). Specifically, there was up to a $45 \%$ decrease in the proportion of cells undergoing EndMT in Endo-Hdac $9^{K O}$ mice in the aortic root, ascending aorta, and aortic arch, despite similar numbers of CD31 ${ }^{+}$endothelial cells (Figure 5 and Supplemental Figure 7) and $\mathrm{CD}^{+} 8^{+}$macrophages (Supplemental Figure 6F).

We next turned to evaluate the atherosclerotic burden and phenotype. En face analysis of thoracic aortas demonstrated that EndoHdac ${ }^{K O}$ mice had a relative $25 \%$ decrease in aortic plaque area compared with control mice (Figure 6A). Consistent with this, oil red $\mathrm{O}$ staining indicated reduced plaque area in Endo- $\mathrm{Hdac} \mathrm{O}^{\mathrm{KO}}$ mice compared with controls in both the aortic root and arch (Figure 6B and Supplemental Figure 8, A and B). Furthermore, plaque lipid content was significantly reduced in the aortic root and ascending aorta of Endo-Hdac $9^{K O}$ mice compared with controls (Figure 6B and Supplemental Figure 8, A and B). Calcification was also significantly reduced in plaques from the aortic root of Endo- $\mathrm{Hac}_{\mathrm{K}} \mathrm{KO}^{\circ}$ mice (Figure 6C), although not in the ascending aorta or aortic arch (Supplemental Figure 8, C and D). In addition, by Masson's trichrome staining of aortic root sections, while the extent of collagen and presence of necrotic cores were similar between Endo- $H d a c 9^{K O}$ mice and controls, there was a significant increase in fibrous cap thickness in Endo-Hdac ${ }^{K O}$ mice (Figure 6D). While there were no differences in these plaque features in the ascending aorta (Supplemental Figure $8 \mathrm{E}$ ), in contrast, in aortic arch plaques, there was a significant increase in plaque collagen content and fibrous cap thickness in Endo-Hdac $9^{K O}$ mice (Supplemental Figure 8F). Intraplaque hemorrhage was not different between Endo- $\mathrm{Hdac} 9^{\mathrm{KO}}$ mice and controls (Figure 6E and Supplemental Figure 9, A and B). Finally, consistent with our ex vivo data (Figure 3D), cell proliferation in the endothelial and subendothelial layers was increased in EndoHdac ${ }^{K O}$ mice compared with controls (Figure 6F and Supplemental Figure 9, C and D). Collectively, these findings indicate that in the setting of atherosclerosis, the absence of $\mathrm{Hdac} 9$ in endothelial cells is associated with reduced EndMT, reduced atherosclerotic burden, and a more favorable plaque phenotype.

Class IIa HDAC inhibition reduces EndMT during atherosclerosis and leads to a more favorable plaque phenotype. Given these findings, as a potential pathway to a clinical therapy, we evaluated the efficacy of class IIa HDAC inhibition using MC1568 in mice with established atherosclerosis. Specifically, $A p o E^{-/-}$mice were placed on a high-fat diet at 8 weeks of age for 18 weeks to induce atherosclerosis. After 10 weeks of high-fat diet, when atherosclerosis was already estab- 
A

A

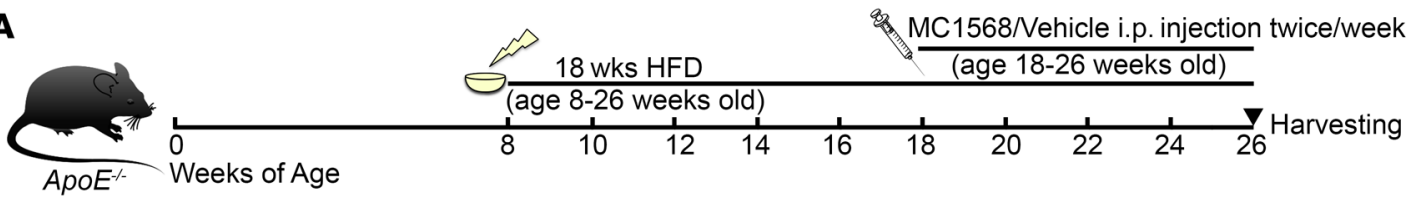

B

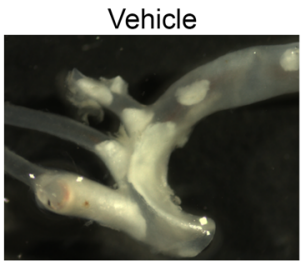

MC1568
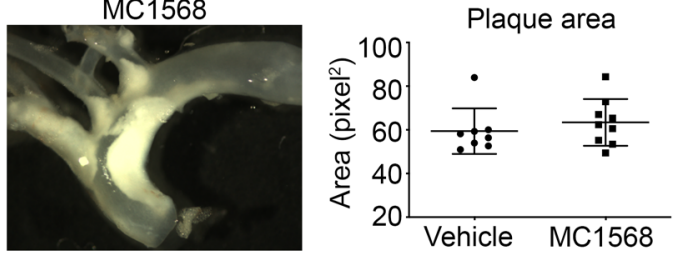

C
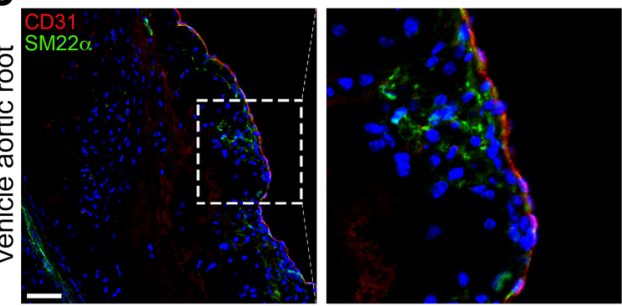

$\mathrm{SM} 22 \alpha^{+} \mathrm{CD} 31^{+}$cells/CD $31^{+}$

CD $31^{+}$cells per image
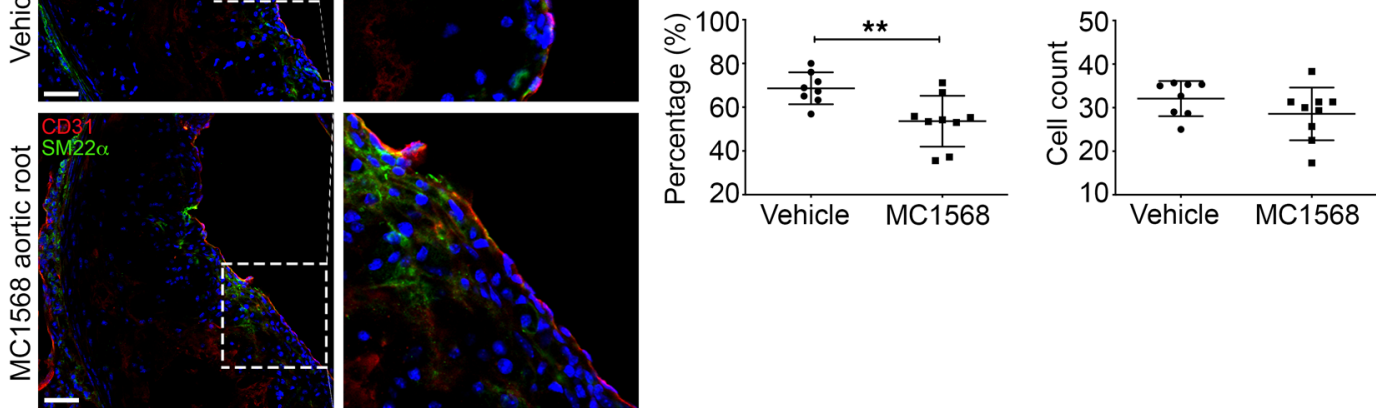

D
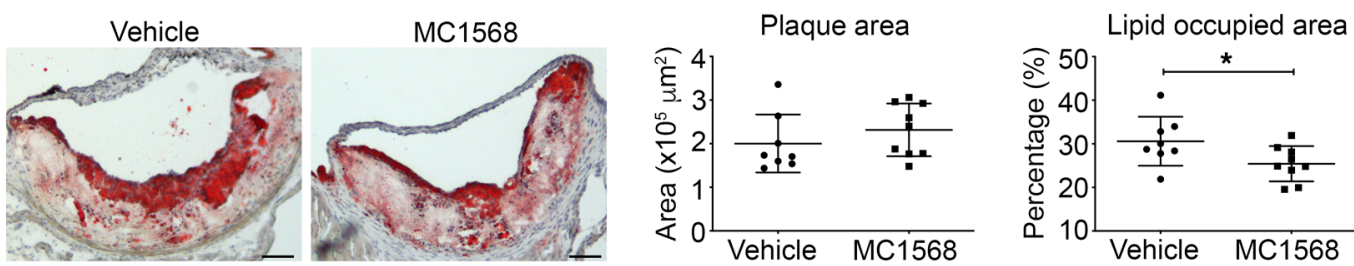

$\mathbf{E}$

Vehicle

MC1568
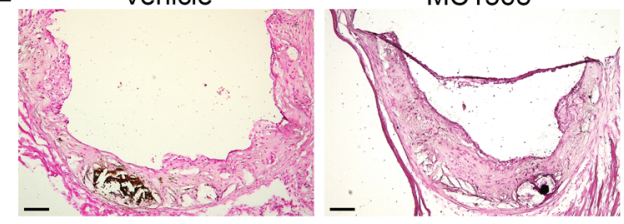

$\mathbf{F}$
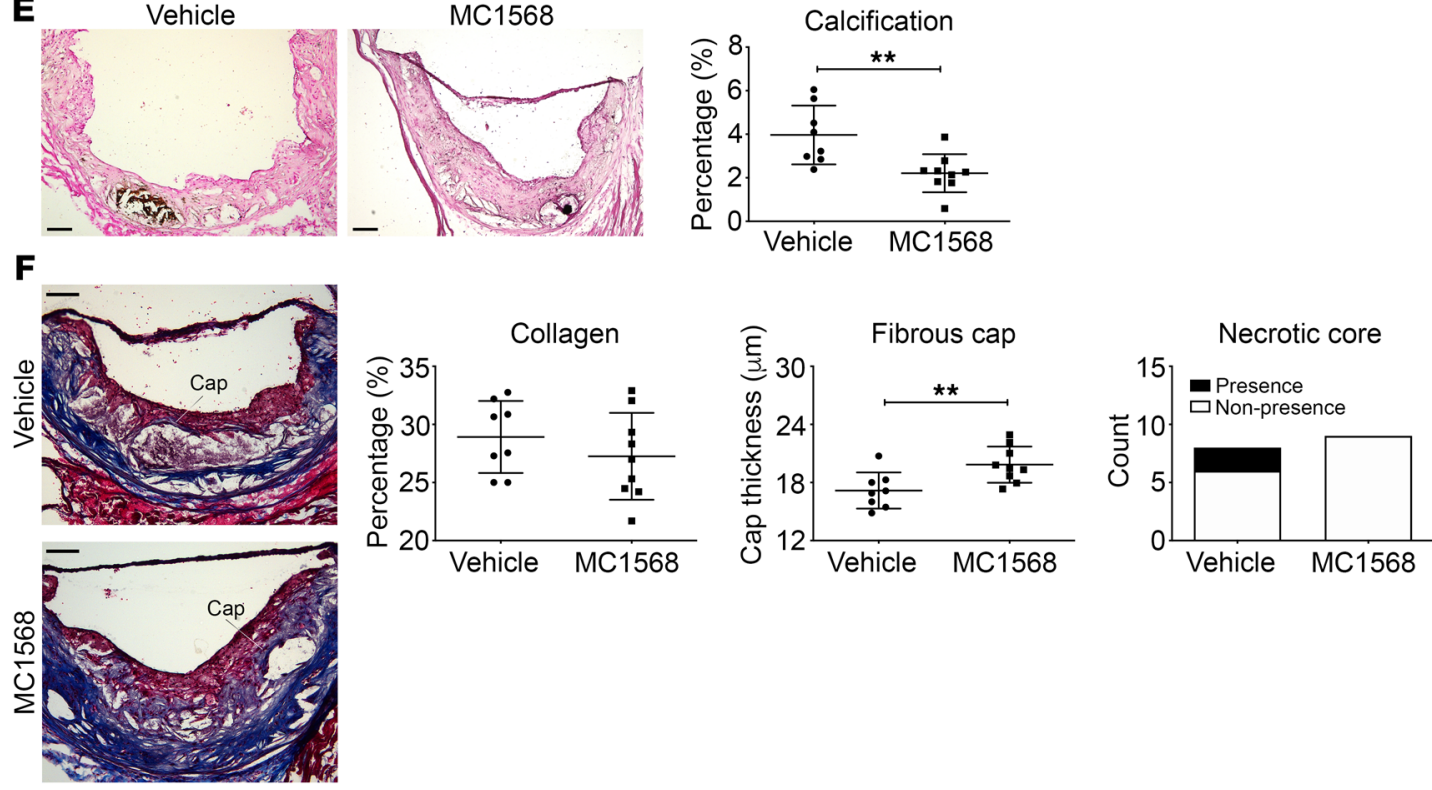
Figure 7. Systemic administration of a class Ila HDAC inhibitor reduces EndMT and increases plaque stability. (A) Generation of MC1568-treated atherosclerotic mouse model. (B) Representative images of aorta before embedding into OCT and en face analysis of plaque in aorta. (C) Representative images of immunofluorescence staining of aortic root sections for SM22 $\alpha$ - (green), CD31- (red), and DAPI-stained nuclei (blue) and quantification of SM22 $\alpha^{+}$ CD31+-costained cells over total CD31+ cells and the total number of CD $31^{+}$cells per image. (D) Representative images of the aortic root with staining using oil red O, with quantification of total plaque area and plaque lipid content. (E) Representative images of aortic root sections using Von Kossa stain (black represents calcification), with quantification of calcification. (F) Representative aortic root images stained with Masson's trichrome with quantification of collagen content, fibrous cap thickness, and presence/nonpresence of necrotic core (blue, collagen; pink, macrophages and cardiac muscle). Scale bars: 50 $\mu \mathrm{m}$ (C); $100 \mu \mathrm{m}$ (D-F). Right panels in C are digital enlargements of the original adjacent images. $n=8$ controls (vehicle) versus $n=9$ MC1568-treated mice for all panels. ${ }^{*} P \leq 0.05$; ${ }^{* *} P \leq 0.01$. All analyses performed using unpaired Student's $t$ test except plaque area (B and $\mathbf{D}$ ), for which Mann-Whitney $U$ test was used. In addition, presence or nonpresence of necrotic core $(\mathbf{F})$ was analyzed using Fisher's exact test.

lished, mice were randomly assigned to receive MC1568 or vehicle by intraperitoneal injection for 8 weeks (until sacrifice and tissue harvest) (Figure 7A). Administration of MC1568 did not alter body weight or lipid profile compared with those in vehicle-administered control mice (Supplemental Figure 10, A and B). However, compared with controls, mice receiving MC1568 showed a reduction in total WBC count, monocyte count, and lymphocyte count. There was no difference in RBC, platelet, or neutrophil counts between groups (Supplemental Figure 10, C and D).

En face analysis of thoracic aortas demonstrated no difference in aortic plaque area in mice that received MC1568 versus vehicle (Figure 7B). EndMT in atherosclerotic plaques was again evaluated by immunofluorescence costaining and confocal imaging for $\mathrm{CD} 31^{+} \mathrm{SM} 22 \alpha^{+}$copositive cells, which demonstrated that class IIa HDAC inhibition by MC1568 was associated with a significant reduction in the proportion of endothelial cells undergoing EndMT in vivo (Figure 7C and Supplemental Figure 10, E and F). Immunofluorescence staining also showed no difference in inflammatory cell content (Supplemental Figure 11, A and B). By histochemical staining, consistent with the en face analysis, administration of MC1568 did not affect plaque area; however, there was a significant reduction in plaque lipid content (Figure 7D and Supplemental Figure 11, C and D). Compared with what occurred in control atherosclerotic mice, MC1568 treatment was also associated with a significant reduction in plaque calcification (Figure 7E). In addition, similarly to what was seen in Endo$\mathrm{Hdac}{ }^{\mathrm{KO}}$ mice, while the extent of collagen and presence of necrotic cores were similar, administration of MC1568 was associated with a significant increase in fibrous cap thickness (Figure 7F).

Collectively, in mice with established atherosclerosis, these results indicate that class IIa HDAC inhibition by systemic MC1568 administration leads to reduced EndMT and favorable changes in atherosclerotic plaque morphology.

\section{Discussion}

There is increasing evidence that EndMT plays an important role in adult cardiovascular diseases $(1,2)$. While our understanding of the epigenetic regulation of EMT is substantial, the epigenetic regulation of EndMT remains relatively unexplored. Our study has shown that EndMT is associated with deacetylation of key histone residues, suggesting that histone modifiers play a major role in regulating EndMT. Further, we show that inhibition of class IIa HDACs can prevent EndMT both in vitro and in vivo. Our data also demonstrate that HDAC9 is the predominant class IIa HDAC responsible for these effects. Specifically, HDAC9 regulates key EndMT-associated molecular changes, including both gene and protein expres- sion of endothelial and mesenchymal cell markers, and also TGF- $\beta$ pathway-associated transcription factors. Furthermore, both class IIa HDAC inhibition and genetic Hdac 9 knockout reversed phenotypic endothelial cell changes that were induced by EndMT and led to favorable changes in atherosclerotic plaques, suggesting potential therapeutic benefit in the treatment of atherosclerosis.

In addition to the above key findings, our results add weight to the understanding that, although major pathways may broadly govern the general phenomena of EMT and EndMT, particular molecular aspects of EMT and EndMT are differentially regulated $(1,2,12,13,44)$. Furthermore, Neumann et al. (18) recently noted that EndMT is associated with changes in $\mathrm{H} 3 \mathrm{~K} 4 \mathrm{me} 3$ methylation of 2 key EndMT-associated genes, which is likely mediated via an interaction between the long noncoding antisense transcript of GATA6 and the epigenetic regulator LOXL2. While not a specific focus of our study, we nevertheless noted a global decrease in the protein level of H3K4me3 with EndMT (Figure 1), and the results of Neumann et al. support our finding that HDACs and epigenetic mechanisms play a key role in regulating EndMT (18).

Atherosclerosis is the most common cause of cardiovascular disease and is a chronic process involving cellular, metabolic, and inflammatory events $(45,46)$. EndMT was recently recognized as a significant contributor to atherosclerosis $(12,13,47)$; however, the mechanisms remain to be defined. In parallel, HDAC9 has been implicated in carotid intima-media thickness (48), largevessel ischemic stroke $(48,49)$, atherosclerosis $(50)$, and atherosclerotic aortic calcification in humans (51). Potentially connecting these observations, our experiments demonstrated that $\mathrm{Hdac} 9$ knockout reduces the extent of EndMT in atherosclerotic mice, correlating with decreased plaque area and a more stable plaque phenotype. Moreover, class IIa HDAC inhibition through MC1568 administration, while not altering atherosclerotic plaque size, was associated with reduced EndMT and features of increased plaque stability. The issue of whether increased fibrous cap thickness is related to reduced EndMT versus a specific effect of HDAC9 is the subject of ongoing studies in our laboratory; however, these findings are consistent with prior research showing that a reduction in EndMT is associated with a beneficial effect on atherosclerosis $(13,52)$ and a more stable plaque phenotype in humans (12). Collectively, we propose that these data suggest a pathological HDAC9/EndMT/atherosclerosis axis. In addition, the fact that therapy with MC1568 was able to reduce EndMT and favorably alter plaque phenotype with established atherosclerosis (after 10 weeks of high-fat feeding in $A p o E^{-/-}$mice) is of translational importance, as it argues for the possibility that therapies targeting EndMT via HDAC inhibition may favorably affect atherosclero- 
sis in humans with established disease. Moreover, the appeal of potentially using HDAC inhibitors as a therapy against atherosclerosis is supported by the fact that there are several HDAC inhibitors with US FDA approval that are in current clinical use for other indications, including the pan-HDAC inhibitor vorinostat and the more selective HDAC inhibitor romidepsin (53).

As well as EndMT and atherosclerosis, our study is also part of a growing body of literature indicating that HDACs are fundamental regulators of endothelial biology and endothelial function. HDAC3 is critical for endothelial cell survival, and an increase in HDAC3 is found in endothelial cells exposed to disturbed flow (54). Knockdown of HDAC3 exacerbates the development of atherosclerosis and causes vessel rupture. HDAC3 is also essential for endothelial cell differentiation, and aberrant HDAC3 splicing can induce EndMT through modulation of the TGF- $\beta$ pathway (55). HDAC5 phosphorylation and nuclear export in endothelial cells can enhance the expression of laminar flow-related atheroprotective genes, such as KLF-2 and eNOS (56). Silencing of HDAC5 is proangiogenic and increases endothelial migration, sprouting, and tubule formation by increasing FGF2 expression (57). HDAC7 is fundamental in maintaining vascular integrity by acting as a repressor of MMP10 (58). Absence of HDAC7 is embryonically lethal, with endothelial cells failing to establish cell-cell junctions, which leads to vessel dilatation and rupture (58). Therefore, although we have provided substantial evidence to indicate a major role for HDAC9 in governing EndMT, we cannot exclude the additional possibility that other HDACs may also regulate certain aspects of EndMT.

In terms of HDAC9, our study showed that an increase of HDAC9 expression has potential detrimental effects in endothelial cells, caused by facilitating the loss of cell junctions and by driving endothelial cells toward a mesenchymal phenotype. In addition to our study, others have also demonstrated that HDAC9 plays an important role in endothelial regulation. For example, HDAC9 expression is increased in endothelial cells during cerebral ischemia/reperfusion injury in rats, promoting endothelial dysfunction through an increased inflammatory response, apoptosis, and cell permeability through decreased cell-cell junction proteins (59). Furthermore, overexpression of HDAC9 increases endothelial sprouting by decreasing expression of the miRNA17-92 cluster, which is known to have antiangiogenic activity (60). Together with our findings, these studies reinforce the fact that HDAC9 is of critical importance for governing endothelial function and pathobiology.

Beyond endothelial biology, HDAC9 has been implicated in other cell types that are important in vascular disease. In mice, it has been shown that HDAC9 suppresses macrophage cholesterol efflux and that transplantation with HDAC9-null bone marrow decreases atherosclerotic lesion size and favors an antiinflammatory macrophage phenotype (61). Increased HDAC9 expression is seen in human thoracic aortic aneurysm tissue and also in cell culture models of genetically perturbed vascular smooth muscle cells where increased HDAC9 is associated with increased MMP production and dysregulation of cytoskeletal genes (62). HDAC9 also facilitates adipocyte differentiation and maturation, while HDAC9 knockdown prevents the detrimental effects of chronic high-fat feeding by improving metabolic homeostasis $(63,64)$.
The class IIa HDAC inhibitor MC1568 (used in this study) has been reported to effectively reduce the severity and incidence of abdominal aortic aneurysms in angiotensin II-infused $A p o E^{-/-}$ mice by attenuating the expression of proinflammatory markers and restoring correct organization of elastin and collagen fibers (36). These studies further demonstrate that HDAC9 plays a key role in regulating the molecular dysfunction apparent in aberrant cellular phenotypes, contributing to vascular pathology.

In conclusion, our study demonstrates a unique role for HDAC9 in contributing to vascular pathology by promoting EndMT, which is associated with increased atherosclerosis and an unstable plaque phenotype. Furthermore, we have shown that selective HDAC inhibition is effective in attenuating EndMT in vitro and in vivo. Together with other studies, our data argue strongly for the existence of a pathological HDAC9/EndMT/ atherosclerosis axis and suggest that targeting of HDAC9 may be beneficial for plaque stabilization or slowing the progression of atherosclerotic disease.

\section{Methods}

Cell culture and treatment. HCAECs and HUVECs (Lonza, C-2585 and C2517A, respectively) were cultured at $37^{\circ} \mathrm{C}$ in $5 \% \mathrm{CO}_{2}$ in endothelial basal medium-2 supplemented with EGM-2MV BulletKit (CC-3202, Lonza) and used at passages 6-7. To induce EndMT, 70,000 cells/well in a 6-well plate or 250,000 cells in a $10 \mathrm{~cm}$ dish were plated and designated day 0. Complete EGM-2MV medium was changed every other day (days 1 and 3 after plating). EndMT was induced by incubating cells in $50 \mathrm{ng} / \mathrm{mL}$ recombinant human TGF- 32 (100-35B, PeproTech) and $200 \mathrm{mM} \mathrm{H}_{2} \mathrm{O}_{2}$ (H1009, MilliporeSigma) added to complete media on days 1 and 3, as described (12). For experiments using MC1568 (16265, Cayman), cells were treated with 1, 3, or $7 \mu \mathrm{M}$ MC1568, 30 minutes prior to the first EndMT induction and then daily for the duration of EndMT. Cells were harvested after 5 days of treatment. For EndMT induction using TGF- $\beta 2+$ IL-1 $\beta$, HUVECs were treated with 10 $\mathrm{ng} / \mathrm{mL}$ TGF- $\beta 2$ and $1 \mathrm{ng} / \mathrm{mL}$ IL-1 $\beta$ daily for 7 days, and as indicated, 7 $\mu \mathrm{M}$ MC1568 was added 30 minutes prior to the addition of cytokines.

MPLECs were obtained from Endo-Hdac $9^{K O}$ mice (Cdh5-CreER ${ }^{T 2}$; $\left.H d a c 9^{f / f}\right)$. For each batch of MPLECs, 4 Endo-Hdac $9^{K O}$ mice were anesthetized by intraperitoneal injection and perfused with $10 \mathrm{~mL}$ icecold HBSS $^{++}$buffer (Supplemental Table 1). Lungs were removed and rinsed in RPMI 1640 medium (11875093, Thermo Fisher) with $1 \%$ antibiotic/antimycotic (15240-112, Life Technologies) before being minced using a scalpel. Minced lungs were digested in $20 \mathrm{~mL} \mathrm{HBSS}^{++}$buffer containing $0.2 \%$ collagenase A (10103586001, MilliporeSigma) for 2 hours at $37^{\circ} \mathrm{C}$ with constant tilting. Cells were then filtered through a $70 \mu \mathrm{m}$ nylon filter (352350, Falcon) and washed twice before resuspension in $3 \mathrm{~mL}$ 0.1\% BSA (A9647, MilliporeSigma) in PBS (used as $1 \times$ PBS in all experiments), which was further incubated with $2.7 \mu \mathrm{g}$ CD31-coated (553370, BD Biosciences) Dynabeads (11035, Thermo Fisher Scientific) for 60 minutes at $4^{\circ} \mathrm{C}$ on a roller. Bead-bound cells were collected using a magnet, resuspended in mouse endothelial cell medium (M1168, Cell Biologics), and cultured for at least 7 days to allow adherence and initial expansion. After further expansion and at passages 2-3, to ablate Hdac9 and induce EndMT in MPLECs, 70,000 cells/well were seeded onto a 6-well plate and designated day 0 . On day 1 , medium was replaced with $1 \mathrm{~mL}$ mouse endothelial cell medium plus $10 \mu \mathrm{M} 4-\mathrm{OH}$ tamoxifen (H7904, MilliporeSigma) or the same vol- 
ume of excipient. 4-OH tamoxifen was reconstituted in ethanol, then diluted with peanut oil (P2144, MilliporeSigma) per the manufacturer's instructions. On days 3 and 5, medium was replaced with mouse endothelial cell medium containing (a) $2 \mu \mathrm{M} 4-\mathrm{OH}$ tamoxifen or equivalent volume of vehicle; and/or (b) $50 \mathrm{ng} / \mathrm{mL}$ of recombinant mouse TGF- $\beta 2$ (CK35, Bon Opus Biosciences) plus $200 \mathrm{mM} \mathrm{H}_{2} \mathrm{O}_{2}$. Cells were harvested for further study on day 7 after plating.

Flow cytometry. Flow cytometry was performed on HCAECs after 5-day EndMT induction, with or without treatment with MC1568 as described above. After trypsinization and washing, cells were fixed for 10 minutes in $4 \%$ PFA and then washed in PBS. Cells were then stained with anti-FAP-Alexa Fluor 647 (bs-5758R-A647, Bioss) or isotype control in PBS containing 0.5\% saponin for 2 hours on ice. Cells were washed with PBS before analysis on a BD LSR II Flow Cytometer.

Animals and treatments. For all in vivo experiments, experimental groups comprised nearly equal numbers of male and female mice, and results are presented in aggregate (males and females combined). Sex-specific data are provided for body weight.

For Hdac 9 knockout experiments, C57BL6 Hdac $9^{f / f l}$ mice (62) were crossed with C57BL6 Cdh5-CreER ${ }^{T 2}$ mice (65) to generate an inducible endothelial-specific Hdac9 knockout model (Cdh5-CreER $\left.{ }^{T 2} ; H d a c 9^{f / f l}\right)$. All mice (Cdh5-CreER $R^{T 2} ; H d a c 9^{f / f l}$ and Hdac $9^{f / f l}$ littermate controls) received $2 \mathrm{mg}$ tamoxifen/d for 5 consecutive days via intraperitoneal injection beginning at 5 weeks of age. To induce atherosclerosis, all mice received a single dose $\left(100 \mu \mathrm{l}\right.$ volume containing $1.5 \times 10^{11}$ virus particles/mouse) of gain-of-function (D377Y) murine PCSK9-encoding recombinant adeno-associated viral vector (rAAV-PCSK9) at 7 weeks of age as described (66). All mice were fed a high-fat adjusted calories diet (42\% from fat, TD88137, Envigo) from 8 weeks of age for 16 weeks. At week 8 (immediately before initiation of high-fat diet), week 16, and week 24 (before intraperitoneal BrdU injection), blood pressure was measured using a CODA noninvasive mouse blood pressure system (Kent Scientific Corp.) according to the manufacturer's instructions. Three blood pressure measurements were performed, and the mean was taken. Tissues were harvested when mice were 24 weeks of age. BrdU (ab142567, Abcam) was dissolved at $10 \mathrm{mg} / \mathrm{mL}$ in sterile PBS and was administered at $10 \mu \mathrm{l} / \mathrm{g}$ of body weight via intraperitoneal injection 24 hours before tissue harvesting. Genotyping of mice was validated by PCR of DNA samples at 3 to 4 weeks of age (Supplemental Table 2).

For in vivo experiments with $\mathrm{MC1568,} \mathrm{8-week-old} A p o E^{-/-}$mice were placed on a high-fat adjusted calories diet for 18 weeks to induce atherosclerosis. After 10 weeks of high-fat diet, mice were randomly assigned to receive MC1568 $(n=9)$ or vehicle $(n=8)$ by intraperitoneal injection for 8 weeks. MC1568 was dissolved in sterile $1 \%$ caboxymethylcellulose (419273, MilliporeSigma) in PBS and administered twice weekly at $40 \mathrm{mg} / \mathrm{kg}$ during the last 8 weeks of high-fat diet. Control mice were administered $1 \%$ carboxymethylcellulose in PBS. Tissues were harvested when mice were 26 weeks of age.

All animals were euthanized by cardiac puncture and exsanguination under isoflurane inhalation anesthesia (NDC 10019-360-40, Baxter Healthcare). Blood collected from each mouse was placed into a heparinized tube and analyzed for complete blood count (CBC). Plasma was collected for lipid panel analysis by centrifuging blood at $4^{\circ} \mathrm{C}, 1000 \mathrm{~g}$, for 10 minutes and stored at $-80^{\circ} \mathrm{C}$. Exsanguinated mice were perfused with $4 \%$ paraformaldehyde (15710, Electron Microscopy Sciences) plus $0.1 \%$ glutaraldehyde in PBS at $3 \mathrm{~mL} / \mathrm{min}$ via the left ventricle for 7 minutes using an infusion pump (BS-300, Braintree Scientific). The heart, aorta, and great vessels were carefully dissected from the surrounding tissues. Aortas were photographed with a Zeiss microscope (Zeiss Stemi 2000-C with AxioCam ERc 5s camera) on the anterior and posterior sides for en face analysis. The aortic root and aorta were then placed in $20 \%$ sucrose in PBS at $4^{\circ} \mathrm{C}$ overnight before embedding in OCT (62550-01; Electron Microscopy Sciences) and stored at $-80^{\circ} \mathrm{C}$. The aortic root and aorta were cryosectioned at $10 \mu \mathrm{m}$ thickness (CM3050S, Leica) onto precleaned glass slides (12550-19; Fisher Scientific) and stored at $-80^{\circ} \mathrm{C}$.

$C B C$ and cholesterol analysis. $\mathrm{CBC}$ analysis was performed by the Comparative Pathology Laboratory at the Icahn School of Medicine at Mount Sinai using an IDEXX ProCyte Dx Hematology Analyzer (IDEXX BioResearch). Cell counts of RBCs, WBCs, platelets, monocytes, lymphocytes, and neutrophils were obtained using standard procedures according to the manufacturer's instructions. Plasma was sent to IDEXX laboratories for chemical testing. Levels of total cholesterol, HDL cholesterol, LDL cholesterol, and triglycerides were measured according to standard procedures using a Catalyst Dx Analyzer (IDEXX BioResearch).

FACS. Endo-Hdac $9^{K O}$ mice $\left(C d h 5-C r e E R^{T 2} ; H d a c 9^{f / f f}\right)$ and $H d a c 9^{f / f l}$ littermate controls received tamoxifen as described above at 5 weeks of age, and tissues were harvested at 8 weeks of age. Aortas were digested as previously described (12). Each heart was digested with 0.8 Wünsch units/mL of Liberase DL (05401160001, Roche) in $1 \mathrm{~mL}$ of serum-free RPMI 1640 medium at $37^{\circ} \mathrm{C}$ for 45 minutes. Lungs

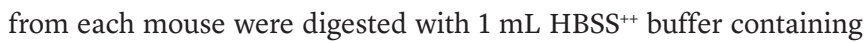
$0.5 \%$ collagenase A at $37^{\circ} \mathrm{C}$ for 45 minutes. Blood was also obtained and treated with RBC lysis buffer (00-4333-57, eBioscience). In brief, $30 \mathrm{~mL}$ ice-cold RBC lysis buffer was added to $2 \mathrm{~mL}$ blood. After incubation for 15 minutes, the mixture was centrifuged for 8 minutes at $400 \mathrm{~g}$. After aspirating the supernatant, another $30 \mathrm{~mL}$ ice-cold RBC lysis buffer was added and the pellet resuspended. After further incubation for 15 minutes, the mixture was again centrifuged for 8 minutes at $400 \mathrm{~g}$. Each sample was finally resuspended in $900 \mu \mathrm{l}$ RPMI 1640 buffer. Cells from all samples were stained with CD45-APC (1800-11, Fisher; $1 \mu \mathrm{g} / \mathrm{million}$ cells), CD31-PE (102408, BioLegend; $1 \mu \mathrm{g} / \mathrm{million}$ cells), and DAPI, followed by sorting using a FACS LSRII flow cytometer (BD Biosciences). qRT-PCR was performed on sorted cells to evaluate $H$ dac 9 levels as described below.

Histological and immunofluorescence analysis of atherosclerotic lesions. Studies were performed in accordance with the 2017 American Heart Association recommendations for animal atherosclerosis studies (67). Frozen sections were thawed at room temperature and rinsed in PBS to remove OCT. For all immunofluorescence staining except as described, tissues were first blocked using 20\% donkey serum (D9663, MilliporeSigma) with 5\% BSA in PBS. For BrdU staining, slides were incubated with prewarmed 1N HCL (1090571000, MilliporeSigma) for 45 minutes at $37^{\circ} \mathrm{C}$ before blocking. Sections were then incubated overnight at $4^{\circ} \mathrm{C}$ with primary antibodies in 3\% BSA in PBS (for antibodies, see Supplemental Table 6). Isotype control slides were incubated with isotype-specific $\operatorname{IgG}$ with the same concentration and species as the primary antibody. Slides were then washed 3 times in PBS and incubated for 1 hour at room temperature with the appropriate secondary antibodies: anti-rat Alexa Fluor 546 at $4.0 \mu \mathrm{g} / \mathrm{mL}$ (catalog A11081, Invitrogen) and/or anti-rabbit Alexa Fluor 488 at $4.0 \mu \mathrm{g} / \mathrm{mL}$ (catalog A21206, Invitrogen). Prior to imaging, slides were washed 3 times with 
PBS and mounted with VECTASHIELD Antifade Mounting Medium with DAPI (H-1200, Vector Laboratories). For immunohistochemical analysis, aortic sections were stained with oil red O (ab150678, Abcam), Masson's trichrome (HT15, MilliporeSigma), and Von Kossa stain (ab150687, Abcam) according to the manufacturer's instructions. Presence of necrotic core was defined as a typically appearing necrotic core occupying $15 \%$ or greater of the total plaque by Masson's trichrome staining (67). Immunohistochemical and CD45 or CD68 images were acquired using a Leica DMi8 microscope, while other immunofluorescence staining was imaged by confocal microscopy (LSM780, Zeiss). All quantification was done blinded, and data were averaged from at least 3 separate plaque sections in aortic root and 2 separate plaque sections in the ascending and arch aorta per mouse. Image $(\mathrm{NIH}$, version 1.52a for Windows) or Fiji software (version 2.0.0-rc-68/1.52w for $\mathrm{Mac}$ ) was used for image analysis and quantification.

HDAC9 staining. For HDAC9 immunofluorescence staining of mouse samples, tissues were fixed with $4 \%$ PFA for 10 minutes and permeabilized with $0.3 \%$ Triton X-100 with PBS for another 10 minutes. Sections were then blocked with 5\% BSA in antibody diluent with background-reducing components (S3022, DAKO) for 1 hour at room temperature, followed by overnight incubation at $4^{\circ} \mathrm{C}$ in antibody diluent with background-reducing components plus $0.1 \%$ Triton $\mathrm{X}-100$ with the following primary antibodies in the indicated combinations: anti-HDAC9, anti-CD31, and/or anti- $\alpha$ SMA (see Supplemental Table 6). Sections were incubated with appropriate secondary antibodies diluted into antibody diluent with background-reducing components and incubated for 1 hour at room temperature. Slides were imaged by confocal microscope (LSM 780, Zeiss).

For HDAC9 immunocytochemistry staining of HUVECs, cells were first cultured under 3 conditions: control, EndMT induction, and EndMT induction plus $7 \mu \mathrm{M}$ MC1568, as described above. HUVECs were fixed with $4 \%$ PFA for 10 minutes, followed by washing with PBS, then permeabilization with $0.3 \%$ Triton X-100 with $\mathrm{PBS}$ for another 10 minutes. HUVECs were next blocked for 30 minutes with $0.1 \%$ Tween-20 in 5\% BSA and then incubated overnight at $4^{\circ} \mathrm{C}$ on a rocker with anti-HDAC9 antibody (catalog ab109446, Abcam). Cells were then washed in PBS at room temperature and incubated with antirabbit Alexa Fluor 488 as secondary antibody (catalog A21206, Invitrogen) in 1\% BSA in PBS. Finally, cells were washed and incubated with DAPI (D3571, Invitrogen) diluted 1:1000 in PBS for 5 minutes. The DAPI-containing solution was then replaced with PBS, and cells were imaged on an inverted microscope (DMi8, Leica).

qRT-PCR. For cells in culture except HUVECs, shown in Supplemental Figure 3, RNA was extracted using an RNeasy Mini Kit according to the manufacturer's instructions (74104, QIAGEN) and stored at $-80^{\circ} \mathrm{C}$ until use. RNA quantity and quality were analyzed using a NanoDrop 2000c Spectrophotometer (Thermo Scientific). Reverse transcription was performed using the iScript cDNA Synthesis Kit (1708891 , Bio-Rad) according to the manufacturer's instructions. The reaction conditions were $25^{\circ} \mathrm{C}$ for 5 minutes, $46^{\circ} \mathrm{C}$ for 20 minutes, $95^{\circ} \mathrm{C}$ for 1 minute, and finally maintained at $4^{\circ} \mathrm{C}$. For cells obtained from FACS (endothelial cells or leukocytes), RNA was extracted using an RNeasy Micro Kit according to the manufacturer's instructions (74004, QIAGEN). cDNA was synthesized and amplified using the qRT-PCR-compatible Ovation RNA-Seq System, version 2 (7102, Tecan Genomics). cDNA quantity and quality were analyzed using a NanoDrop 2000c Spectrophotometer. For all cell types except HUVECs, shown in Sup- plemental Figure 3, qRT-PCR was performed using the PerfeCTa SYBR Green FastMix Reaction Kit according to the manufacturer's instructions (101414-292, VWR) and was performed at $95^{\circ} \mathrm{C}$ for 5 minutes, followed by 40 cycles of $95^{\circ} \mathrm{C}$ for 5 seconds and $60^{\circ} \mathrm{C}$ for 30 seconds. $18 \mathrm{~S}$ rRNA was used as a control and gene expression analyzed using the $\Delta \Delta \mathrm{Ct}$ method. Primers are listed in Supplemental Tables 3 and 4.

For HUVECs shown in Supplemental Figure 3, except for HDAC9, qRT-PCR was performed using TaqMan Gene Expression probes. RNA isolation, reverse transcription, and qRT-PCR were performed as described in Monteiro et al. (68). For detection of HDAC9 in HUVECs, qRT-PCR was performed using the PerfeCTa SYBR Green FastMix Reaction Kit as described above. Human ubiquitin protein C (UBC) was used for qRT-PCR normalization in Supplemental Figure 3.

ChIP-qPCR. Total chromatin extraction was performed as previously described (69) with the following modifications. Briefly, cells were lysed in buffer A (10 mM HEPES pH = 7.9, $10 \mathrm{mM} \mathrm{KCl,} 1.5 \mathrm{mM}$ $\mathrm{MgCl}_{2}, 0.34 \mathrm{M}$ sucrose, $10 \%$ glycerol, inhibitor cocktail: $1 \mathrm{mM}$ DTT, $0.1 \%$ Triton $\mathrm{X}-100)$ and incubated for 8 minutes on ice. Nuclear pellets were obtained by centrifugation at $1,500 \mathrm{~g}$ for 5 minutes at $4^{\circ} \mathrm{C}$, washed twice with buffer A, and placed into Douncing buffer $(10 \mathrm{mM}$ Tris, $4 \mathrm{mM} \mathrm{MgCl}_{2}, 1 \mathrm{mM} \mathrm{CaCl}_{2}, \mathrm{pH}$ 7.5). Pellets of unfixed nuclei were processed for native-ChIP as previously described (70) starting from step 30 with the following modifications. ChIP was performed using 4 $\mu \mathrm{l}$ anti-H3K27ac antibody, $4 \mu \mathrm{l}$ anti-H3K9ac antibody, or $4 \mu \mathrm{l}$ anti-IgG antibody. ChIP-qPCR was performed using the Perfecta SYBR Green FastMix Reaction Kit according to the manufacturer's instructions (101414-292, VWR). ChIP-qPCR was performed at $95^{\circ} \mathrm{C}$ for 5 minutes followed by 40 cycles of $95^{\circ} \mathrm{C}$ for 5 seconds and $60^{\circ} \mathrm{C}$ for 30 seconds using primers corresponding to the promoter region of each gene of interest listed in Supplemental Table 5. qRT-PCR was performed in triplicate, and the percentage of input was calculated. Data are representative of $n=4$ independent experiments.

Histone PTM and protein Western blotting. For histone PTM quantification, chromatin was obtained as previously described (69) with the following modifications. Final chromatin pellets were resuspended in Laemmli sample buffer (161-0737, Bio-Rad) plus 5\% $\beta$-mercaptoethanol and boiled for 5 minutes at $95^{\circ} \mathrm{C}$. Histones were separated by SDS-PAGE gel electrophoresis, transferred to polyvinylidene fluoride membrane (PVDF, 88520, Thermo Fisher Scientific), and stained using amido black staining solution (A8181, MilliporeSigma) to confirm equal loading. Membranes were blocked with $5 \%$ skim milk powder in TBS-t for 1 hour at room temperature.

For protein extraction, cells were collected into RIPA buffer (89900, Thermo Scientific) with protease and phosphatase inhibitor (78441, Thermo Scientific) on ice and lysed using a BD Lo-Dose insulin syringe (14-826-79, Fisher Scientific). Protein concentration was measured using a Pierce BCA Protein Assay Kit (23255, Thermo Fisher Scientific) according to the manufacturer's instructions using a SpectraMax M5 microplate reader (Molecular Devices). Ten micrograms of protein was loaded and separated by SDS-PAGE gel electrophoresis, transferred to PVDF membrane, and blocked in 5\% BSA for 1 hour at room temperature.

Primary antibodies were incubated overnight at $4^{\circ} \mathrm{C}$ as indicated in Supplemental Table 6. Membranes were washed in TBS-t and incubated in goat anti-rabbit IgG $(\mathrm{H}+\mathrm{L})$ secondary antibody, HRP (diluted 1:15,000, catalog 31460, ThermoFisher Scientific), or goat anti-mouse IgG (H+L) secondary antibody, HRP (diluted 1:15,000, catalog 31430, 
ThermoFisher Scientific) for 1 hour at room temperature. Membranes were visualized using Immobilon Western chemiluminescence HRP substrate (WBKLS0500; EMD Millipore) and imaged on a ChemiDoc Touch Imaging System (Bio-Rad). The full, uncut gels can be found in the supplemental material. Densitometry analysis was performed using Image Lab 5.2 software from Bio-Rad.

Cell fractionation. Cells $\left(5 \times 10^{6}\right)$ were collected and washed twice with cold PBS. The cell pellet was transferred into a prechilled microcentrifuge tube and gently resuspended and incubated for 15 minutes with $500 \mu \mathrm{L} 1 \times$ hypotonic buffer (20 mM Tris- $\mathrm{HCl} 7.5,10 \mathrm{mM} \mathrm{NaCl}, 3 \mathrm{mM} \mathrm{MgCl}_{2}$, with protease and phosphatase inhibitor). Then, $25 \mu \mathrm{l} 10 \% \mathrm{NP} 40$ was added and vortexed for 10 seconds. The homogenate was centrifuged at $4^{\circ} \mathrm{C}$ for 10 minutes at $1,200 \mathrm{~g}$, and the supernatant (cytoplasmic fraction) was removed and stored at $-80^{\circ} \mathrm{C}$. The nuclear pellet was washed once with $250 \mu \mathrm{L} 1 \times$ hypotonic buffer with protease and phosphatase inhibitor. The nuclear pellet was then resuspended in $50 \mu \mathrm{L}$ RIPA buffer for 30 minutes on ice with vortexing at 10-minute intervals. The homogenate was centrifuged at $4^{\circ} \mathrm{C}$ for 30 minutes at $14,000 \mathrm{~g}$. The supernatant (nuclear fraction) was stored at $-80^{\circ} \mathrm{C}$ prior to Western blotting.

Crystal violet and TUNEL assay. HCAECs at a density of 18,000 cells/well or MPLECs at 25,000 cells/well were seeded onto 24-well plates. HCAECs were treated with or without EndMT and with or without MC1568 as described above. MPLECs were treated with 4-OH tamoxifen or vehicle and with or without EndMT, as described above (quantities of reagents and media were scaled to 24-well format). Cells were then fixed with ice-cold $4 \%$ PFA for 10 minutes and stained with $0.5 \%$ crystal violet (C3886, MilliporeSigma) for another 10 minutes before washing with tap water. Images were taken with an inverted microscope (DMi8, Leica). For TUNEL assay, HCAECs or MPLECs were cultured and fixed as described for the crystal violet assay. An In Situ Cell Death Detection Kit (12156792910, MilliporeSigma) was used according to the manufacturer's instructions.

Cell proliferation assay. For HCAECs, cells were plated onto a 96-well plate at 3000 cells/well in replicates of 5 and EndMT induced as indicated above. After 24 hours, a BrdU Cell Proliferation ELISA Colorimetric Kit (11647229001, Roche) was used according to the manufacturer's instructions. BrdU was applied for 10 hours, and absorbance was measured at $370 \mathrm{~nm}$ using a SpectraMax M5 microplate reader. The average of 5 experimental replicates was used to calculate the average proliferation rate per HCAEC line.

For MPLECs, cells were treated with 4-OH tamoxifen or vehicle for 2 days and then also with or without EndMT induction for another 2 days ( 4 treatment conditions in total). Cell were then trypsinized and plated onto a 96-well plate at 3000 cells/well in replicates of 3 and were cultured for a further 2 days with or without induction of EndMT (without 4-OH tamoxifen or vehicle). A BrdU Cell Proliferation Assay Kit (K306-200; BioVision) was then used according to the manufacturer's instructions. In brief, BrdU was incubated for 4 hours. Absorbance was measured at $650 \mathrm{~nm}$ using a SpectraMax M5 microplate reader $25 \mathrm{~min}$ utes after TMB substrate was added. The average of 3 experimental replicates was used to measure proliferation for each biological replicate.

Tubule-formation assay. For HCAECs, cells underwent EndMT induction for 5 days with or without MC1568. MPLECs were treated with 4-OH tamoxifen or vehicle and with or without EndMT, as described above. Cells were then trypsinized, counted, and plated onto growth factor-free Matrigel (CB-40230A, Fisher Scientific) coated onto a 96-well plate at 6000 cells/well. Cells were replaced into the incubator for 4 hours before imaging. Two images/well were captured at $\times 10$ magnification using phase-contrast microscopy. Tubule-branching points were counted using ImageJ software.

Contraction assay. For HCAECs, cells underwent EndMT induction for 5 days with or without MC1568. MPLECs were treated with 4-OH tamoxifen or vehicle and with or without EndMT, as described above. Thereafter, cells were trypsinized and resuspended in complete medium at $1 \times 10^{6}$ cells $/ \mathrm{mL}$. Cell suspensions were mixed 1:4 with a collagen gel working solution according to the manufacturer's protocol (CBA-201, Cell Biolabs) and plated onto 48-well plates using 250 $\mu \mathrm{L} /$ well and allowed to set for 1 hour. After the collagen was set, 500 $\mu \mathrm{L}$ serum-free medium was loaded into each well for 1 hour to serum starve the cells before replacing $400 \mu \mathrm{L}$ of serum-free medium with complete medium plus lysophosphatidic acid (LPA) (catalog 3854, R\&D Systems; contraction initiator, final concentration at $10 \mu \mathrm{M})$, and the gels were then released. Gels were imaged after 24 hours and contraction measured by calculating gel area using Image J software.

Exogenous HDAC9 overexpression. HUVECs were seeded into 10 $\mathrm{cm}$ dishes. After 24 hours, cells were serum starved for 1 hour prior to transfection with Lipofectamine 3000 transfection reagent according to the manufacturer's instructions (Thermo Fisher Scientific; L3000008). Cells were transfected with Flag-tagged full-length HDAC9 cDNA construct (71) cloned in a pCMV-3tag6 vector (Agilent Technologies) or empty vector as a control. Cells were harvested at 24 hours, 48 hours, and 72 hours after transfection and tested for exogenous HDAC9 expression. HDAC9 mRNA was detected by qRT-PCR with primers shown in Supplemental Table 3, and HDAC9 protein was detected by Western blotting using an anti-HDAC9 primary antibody and anti-Flag antibody, as listed in Supplemental Table 6. Exogenous HDAC9 localization was detected using anti-Flag primary antibody after cells were fixed in $4 \%$ paraformaldehyde.

Statistics. No outliers or data points were excluded from this study. For all HCAEC experiments, 3 to 8 different donors were used as biological replicates and all data were acquired through a minimum of 3 independently performed experiments. For all HUVEC and MPLEC experiments, a minimum of 3 independent experiments were performed. For in vitro and ex vivo data, unpaired Student's $t$ test, 1-way ANOVA, or 2-way ANOVA with post hoc Tukey's multiple comparison test was used as stated. For in vivo data, we first tested each data set using the Shapiro-Wilk test to assess for normality of distribution. For normally distributed in vivo data, we applied an unpaired Student's $t$ test. For in vivo data that were not normally distributed, we applied a Mann-Whitney $U$ test. A single exception for the in vivo data was for considering the presence or nonpresence of necrotic core, where, due to the categorical nature of these data, Fisher's exact test was used. The specific test applied for each comparison is stated in each figure legend. Statistical analyses were performed using Prism 8 or 9 , and a 2 -sided $P$ value of less than 0.05 was considered significant. All data are presented as mean $\pm \mathrm{SD}$.

Study approval. All mice were housed in the animal facility at Icahn School of Medicine at Mount Sinai. Animal experiments and protocols were approved by Institutional Animal Care and Use Committee of Icahn School of Medicine at Mount Sinai.

\section{Author contributions}

LL and YX conceived, performed, and analyzed experiments, prepared figures, and cowrote the manuscript. BV performed histo- 
chemical staining and helped with mouse genotyping and harvesting. NC, VDE, MPS, and LM helped with and provided advice on in vitro and in vivo experiments and data. VP performed ChIP-qPCR experiments. DKC and PAG performed HDAC9 pCMV-3tag6 vector cloning. $\mathrm{AC}$ and $\mathrm{AHB}$ performed EndMT in vitro experiments in HUVECs and analyzed these data. MMB and JFB provided rAAV-PCSK 9 and advised on in vivo experiments. HWK and NLW provided pCMV-3tag6-HDAC9 and Hdac $9^{f / f l}$ mice and advised on the use of these resources. SK, MB, VF, and JLMB conceived and supervised several analyses and assisted with manuscript editing. EB provided antibodies and advice for histone PTM quantification, assisted with designing experiments, and helped edit the manuscript. JCK conceived the study, supervised experiments and data analysis, and cowrote the manuscript. All authors edited the manuscript.

\section{Acknowledgments}

This study was directly supported by NIH grant R01HL130423. Confocal microscopy was performed at the Microscopy CoRE and flow cytometry at the Flow Cytometry CoRE of the Icahn
School of Medicine at Mount Sinai. JCK acknowledges support from the NIH (R01HL130423, R01HL135093, R01HL148167). YX was supported by a generous gift from the Haver Foundation and Maurine Haver. NC and VDE were supported by NIH grant T32HL007824. PAG was supported by a Canadian Institutes of Health Research postdoctoral fellowship. JLMB acknowledges support from Astra-Zeneca, NIH R01HL125863, and the Fondation Leducq. NLW is supported by HL142097, HL134354, HL126949, and AR070029. The CNIC is supported by the Instituto de Salud Carlos III (ISCIII), the Ministerio de Ciencia e Innovación (MCIN), and the Pro CNIC Foundation, and is a Severo Ochoa Center of Excellence (SEV-2015-0505). AHB is supported by the BHF Chair of Translational Cardiovascular Sciences and grants RG/14/3/30706 and ERC Advanced Grant VASCMIR. EB acknowledges support from NIH R01CA154683.

Address correspondence to: Jason Kovacic, Icahn School of Medicine at Mount Sinai, One Gustave L. Levy Place, Box 1030, New York, New York 10029, USA. Phone: 212.241.7014; Email: jason. kovacic@mountsinai.org.
1. Kovacic JC, et al. Endothelial to mesenchymal transition in cardiovascular disease: JACC state-of-the-art review. J Am Coll Cardiol. 2019;73(2):190-209.

2. Kovacic JC, et al. Epithelial-to-mesenchymal and endothelial-to-mesenchymal transition: from cardiovascular development to disease. Circulation. 2012;125(14):1795-1808.

3. Piera-Velazquez S, Jimenez SA. Molecular mechanisms of endothelial to mesenchymal cell transition (EndoMT) in experimentally induced fibrotic diseases. Fibrogenesis Tissue Repair. 2012;5(Suppl 1):S7.

4. Hashimoto N, et al. Endothelial-mesenchymal transition in bleomycin-induced pulmonary fibrosis. Am J Respir Cell Mol Biol. 2010;43(2):161-172.

5. Sohal SS. Endothelial to mesenchymal transition (EndMT): an active process in chronic obstructive pulmonary disease (COPD)? Respir Res. 2016;17:20.

6. He J, et al. Role of the endothelial-to-mesenchymal transition in renal fibrosis of chronic kidney disease. Clin Exp Nephrol. 2013;17(4):488-497.

7. Zeisberg EM, et al. Fibroblasts in kidney fibrosis emerge via endothelial-to-mesenchymal transition. J Am Soc Nephrol. 2008;19(12):2282-2287.

8. Zeisberg EM, et al. Endothelial-to-mesenchymal transition contributes to cardiac fibrosis. Nat Med. 2007;13(8):952-961.

9. Jeong D, et al. Matricellular protein CCN5 reverses established cardiac fibrosis. JAm Coll Cardiol. 2016;67(13):1556-1568.

10. Cooley BC, et al. TGF- $\beta$ signaling mediates endothelial-to-mesenchymal transition (EndMT) during vein graft remodeling. Sci Transl Med. 2014;6(227):227ra34.

11. Moonen JR, et al. Endothelial-to-mesenchymal transition contributes to fibro-proliferative vascular disease and is modulated by fluid shear stress. Cardiovasc Res. 2015;108(3):377-386.

12. Evrard SM, et al. Endothelial to mesenchymal transition is common in atherosclerotic lesions and is associated with plaque instability. Nat Commun. 2016;7:11853.

13. Chen PY, et al. Endothelial-to-mesenchymal transition drives atherosclerosis progression. J Clin Invest. 2015;125(12):4514-4528.

14. Dejana E, et al. The molecular basis of endothelial cell plasticity. Nat Commun. 2017;8:14361.

15. Chen PY, et al. FGF regulates TGF- $\beta$ signaling and endothelial-to-mesenchymal transition via control of let-7 miRNA expression. Cell Rep. 2012;2(6):1684-1696.

16. Chen PY, et al. Fibroblast growth factor receptor 1 is a key inhibitor of TGF $\beta$ signaling in the endothelium. Sci Signal. 2014;7(344):ra90.

17. Correia AC, et al. FGF2 inhibits endothelialmesenchymal transition through microRNA-20amediated repression of canonical TGF- $\beta$ signaling. JCell Sci. 2016;129(3):569-579.

18. Neumann P, et al. The lncRNA GATA6-AS epigenetically regulates endothelial gene expression via interaction with LOXL2. Nat Commun. 2018;9(1):237.

19. Terashima M, et al. Epigenetic regulation of epithelial-mesenchymal transition by KDM6A histone demethylase in lung cancer cells. Biochem Biophys Res Commun. 2017;490(4):1407-1413.

20. Pelaez IM, et al. Oncogenic RAS alters the global and gene-specific histone modification pattern during epithelial-mesenchymal transition in colorectal carcinoma cells. Int J Biochem Cell Biol. 2010;42(6):911-920.

21. Wu CY, et al. Epigenetic reprogramming and post-transcriptional regulation during the epithelial-mesenchymal transition. Trends Genet. 2012;28(9):454-463.

22. Serrano-Gomez SJ, et al. Regulation of epithelialmesenchymal transition through epigenetic and post-translational modifications. Mol Cancer. 2016;15:18

23. Seto E, Yoshida M. Erasers of histone acetylation: the histone deacetylase enzymes. Cold Spring Harb Perspect Biol. 2014;6(4):a018713.
24. Dokmanovic M, et al. Histone deacetylase inhibitors: overview and perspectives. Mol Cancer Res 2007;5(10):981-989.

25. Chen X, et al. The epigenetic modifier trichostatin A, a histone deacetylase inhibitor, suppresses proliferation and epithelial-mesenchymal transition of lens epithelial cells. Cell Death Dis. 2013;4:e884

26. Xie L, et al. Histone deacetylase inhibitors trichostatin A and vorinostat inhibit TGF $\beta 2$-induced lens epithelial-to-mesenchymal cell transition. Invest Ophthalmol Vis Sci. 2014;55(8):4731-4740.

27. Wang Y, Shang Y. Epigenetic control of epithelial-to-mesenchymal transition and cancer metastasis. Exp Cell Res. 2013;319(2):160-169.

28. Zheng XX, et al. Histone deacetylases and atherosclerosis. Atherosclerosis. 2015;240(2):355-366.

29. Zhou B, et al. Role of histone deacetylases in vascular cell homeostasis and arteriosclerosis. Cardiovasc Res. 2011;90(3):413-420.

30. Kim J, et al. Restoration of impaired endothelial myocyte enhancer factor 2 function rescues pulmonary arterial hypertension. Circulation. 2015;131(2):190-199.

31. Pang M, Zhuang S. Histone deacetylase: a potential therapeutic target for fibrotic disorders. JPharmacol Exp Ther. 2010;335(2):266-272.

32. Cao DJ, et al. Histone deacetylase (HDAC) inhibitors attenuate cardiac hypertrophy by suppressing autophagy. Proc Natl Acad Sci US A. 2011;108(10):4123-4128.

33. Hwang JY, et al. Epigenetic mechanisms in stroke and epilepsy. Neuropsychopharmacology. 2013;38(1):167-182.

34. Findeisen HM, et al. Epigenetic regulation of vascular smooth muscle cell function in atherosclerosis. Curr Atheroscler Rep. 2013;15(4):319.

35. Usui $\mathrm{T}$, et al. Histone deacetylase 4 controls neointimal hyperplasia via stimulating proliferation and migration of vascular smooth muscle cells. Hypertension. 2014;63(2):397-403. 
36. Galan M, et al. Induction of histone deacetylases (HDACs) in human abdominal aortic aneurysm: therapeutic potential of HDAC inhibitors. Dis Model Mech. 2016;9(5):541-552.

37. Fleming CL, et al. Improved synthesis and structural reassignment of MC1568: a class IIa selective HDAC inhibitor. JMed Chem. 2014;57(3):1132-1135.

38. Mai A, et al. Class II (IIa)-selective histone deacetylase inhibitors. 1. Synthesis and biological evaluation of novel (aryloxopropenyl)pyrrolyl hydroxyamides. JMed Chem. 2005;48(9):3344-3353.

39. Mai A, et al. Identification of two new synthetic histone deacetylase inhibitors that modulate globin gene expression in erythroid cells from healthy donors and patients with thalassemia. Mol Pharmacol. 2007;72(5):1111-1123.

40. Nebbioso A, et al. Selective class II HDAC inhibitors impair myogenesis by modulating the stability and activity of HDAC-MEF2 complexes. EMBO Rep. 2009;10(7):776-782.

41. Scognamiglio A, et al. HDAC-class II specific inhibition involves HDAC proteasome-dependent degradation mediated by RANBP2. Biochim Biophys Acta. 2008;1783(10):2030-2038.

42. Guida N, et al. MC1568 inhibits thimerosalinduced apoptotic cell death by preventing HDAC4 up-regulation in neuronal cells and in rat prefrontal cortex. Toxicol Sci. 2016;154(2):227-240.

43. Moonen JR, et al. Endothelial progenitor cells give rise to pro-angiogenic smooth muscle-like progeny. Cardiovasc Res. 2010;86(3):506-515.

44. Cook DP, Vanderhyden BC. Context specificity of the EMT transcriptional response. Nat Commun. 2020;11(1):2142.

45. Tabas I, et al. Recent insights into the cellular biology of atherosclerosis. J Cell Biol. 2015;209(1):13-22.

46. Moore KJ, et al. Macrophage trafficking, inflammatory resolution, and genomics in atherosclerosis: JACC macrophage in CVD series (Part 2). JAm Coll Cardiol. 2018;72(18):2181-2197.

47. Wesseling M, et al. The morphological and molecular mechanisms of epithelial/endothelialto-mesenchymal transition and its involve- ment in atherosclerosis. Vascul Pharmacol. 2018;106:1-8.

48. Markus HS, et al. Evidence HDAC9 genetic variant associated with ischemic stroke increases risk via promoting carotid atherosclerosis. Stroke. 2013;44(5):1220-1225.

49. International Stroke Genetics Consortium (ISGC), et al. Genome-wide association study identifies a variant in HDAC9 associated with large vessel ischemic stroke. Nat Genet. 2012;44(3):328-333.

50. Azghandi S, et al. Deficiency of the stroke relevant HDAC9 gene attenuates atherosclerosis in accord with allele-specific effects at 7p21.1. Stroke. 2015;46(1):197-202.

51. Malhotra R, et al. HDAC9 is implicated in atherosclerotic aortic calcification and affects vascular smooth muscle cell phenotype. Nat Genet. 2019;51(11):1580-1587.

52. Li A, et al. Endothelial-to-mesenchymal transition: a potential mechanism for atherosclerosis plaque progression and destabilization. DNA Cell Biol. 2017;36(11):883-891.

53. Ganesan A, et al. The timeline of epigenetic drug discovery: from reality to dreams. Clin Epigenetics. 2019;11(1):174.

54. Zampetaki A, et al. Histone deacetylase 3 is critical in endothelial survival and atherosclerosis development in response to disturbed flow. Circulation. 2010;121(1):132-142.

55. Zeng L, et al. Histone deacetylase 3 unconventional splicing mediates endothelial-to-mesenchymal transition through transforming growth factor $\beta 2$. J Biol Chem. 2013;288(44):31853-31866.

56 . Wang W, et al. Fluid shear stress stimulates phosphorylation-dependent nuclear export of HDAC5 and mediates expression of KLF2 and eNOS. Blood. 2010;115(14):2971-2979.

57. Urbich C, et al. HDAC5 is a repressor of angiogenesis and determines the angiogenic gene expression pattern of endothelial cells. Blood. 2009;113(22):5669-5679.

58. Chang S, et al. Histone deacetylase 7 maintains vascular integrity by repressing matrix metalloproteinase 10. Cell. 2006;126(2):321-334.
59. Shi W, et al. HDAC9 exacerbates endothelial injury in cerebral ischaemia/reperfusion injury. JCell Mol Med. 2016;20(6):1139-1149.

60. Kaluza D, et al. Histone deacetylase 9 promotes angiogenesis by targeting the antiangiogenic microRNA-17-92 cluster in endothelial cells. Arterioscler Thromb Vasc Biol. 2013;33(3):533-543.

61. Cao Q, et al. Histone deacetylase 9 represses cholesterol efflux and alternatively activated macrophages in atherosclerosis development. Arterioscler Thromb Vasc Biol. 2014;34(9):1871-1879.

62. Lino Cardenas CL, et al. An HDAC9-MALAT1BRG1 complex mediates smooth muscle dysfunction in thoracic aortic aneurysm. Nat Commun. 2018;9(1):1009.

63. Chatterjee TK, et al. Role of histone deacetylase 9 in regulating adipogenic differentiation and high fat diet-induced metabolic disease. Adipocyte. 2014;3(4):333-338.

64. Chatterjee TK, et al. Histone deacetylase 9 is a negative regulator of adipogenic differentiation. J Biol Chem. 2011;286(31):27836-27847.

65. Wang Y, et al. Ephrin-B2 controls VEGF-induced angiogenesis and lymphangiogenesis. Nature. 2010;465(7297):483-486.

66. Bjorklund MM, et al. Induction of atherosclerosis in mice and hamsters without germline genetic engineering. Circ Res. 2014;114(11):1684-1689.

67. Daugherty A, et al. Recommendation on design, execution, and reporting of animal atherosclerosis studies: a scientific statement from the American Heart Association. Arterioscler Thromb Vasc Biol. 2017;37(9):e131-e157.

68. Monteiro JP, et al. MIR503HG loss promotes endothelial-to-mesenchymal transition in vascular disease. Circ Res. 2021;128(8):1173-1190.

69. Torrente MP, et al. Proteomic interrogation of human chromatin. PLoS One. 2011;6(9):e24747.

70. Kundakovic M, et al. Practical guidelines for high-resolution epigenomic profiling of nucleosomal histones in postmortem human brain tissue. Biol Psychiatry. 2017;81(2):162-170

71. Petrie K, et al. The histone deacetylase 9 gene encodes multiple protein isoforms. J Biol Chem. 2003;278(18):16059-16072. 